\title{
Aspects Regarding Raptors Migration over the Black Sea
}

\author{
Cătălin-Răzvan STANCIU' ${ }^{1}$, Răzvan ZAHARIA², \\ Gabriel-Bogdan CHIȘAMERA4, Ioana COBZARU³, Viorel-Dumitru GAVRIL ${ }^{3,1, *}$, \\ Răzvan-Valentin POPESCU-MIRCENI², Dumitru MURARIU ${ }^{3}$
}

\begin{abstract}
${ }^{1}$ Faculty of Biology, University of Bucharest, 91-95 Splaiul Independenței, 050095 Bucharest, Romania ${ }^{2}$ Oceanographic Research and Marine Environment Protection Society Oceanic-Club, Constanța, Romania ${ }^{3}$ Institute of Biology Bucharest of Romanian Academy, 296 Splaiul Independenței, 060031 Bucharest, Romania

4"Grigore Antipa" National Museum of Natural History, 1 Kiseleff Blvd, 011341 Bucharest, Romania "corresponding author, email: viorel.gavril@ibiol.ro
\end{abstract}

Received: August 2, 2017; Accepted: August 30, 2017; Available online: August 31, 2017; Printed: December 31, 2017

\begin{abstract}
We investigated diurnal raptors movements in the Black Sea basin. Bird migration over the western Black Sea has not been studied properly, scarce data being available mainly by Drost's paper (1930) thus referring only to the birds from Snakes Island, and few new data collected during more recent research expeditions. The study of migration over large water bodies is a very complex task, due to the difficulty of reaching these areas; observations in this paper were made from research vessels and offshore oil rigs. Our data together with previously published information provides a new insight into bird migration routes over the Black Sea. Species observed across the Black Sea basin followed routes that are part of Via Pontica Corridor and Trans-Caucasian Corridor. The aim of this paper is to have a clearer image of the migration phenomenon in this area, such data are important for management and conservation of migratory birds.
\end{abstract}

Key words: bird migration, diurnal raptors, Black Sea route, Via Pontica, Dobruja, Romania.

\section{INTRODUCTION}

The Eurasian - East African flyway is one of the main migration corridor connecting northeastern Europe and western Siberia with southern Africa, through Middle East. It is well known that during their migrations, birds are natural barriers using geographical bottlenecks, Rudescu (1958), Munteanu (1969), Mătieş (1986), etc. Romania and the Black Sea are crossed by one important component of this major flyway, which was named "the Black Sea route". These migration routes have their origin in the north of Aral Lake and of the Caspian Sea, and have an well marked individuality even if, before reaching Dobruja, some of its secondary branches cross the Black Sea to Turkey (Munteanu, 1969).

Over the time, numerous works have been published on the avifauna of coastal Dobruja, including observation about bird migration along the Black Sea coastline, starting with early works of the late $19^{\text {th }}$ century by Simpson (1861), Seebohm (1881), Alleon (1886), Almasy (1898), Sintenis (1877), to early $20^{\text {th }}$ century by Licherdopol (1900), Heinrich (1927), Müller (1926), Kornis (1931), Spiss (1932, 1933) and to more recent papers of Papadopol (1955, 1956, 1963, 1968, 1970), Kummerloewe (1956, 1957a, b), Viellard (1967), Impe (1968, 1969a, b, c), Ciochia (1969, 1970, 1971, 1972), Munteanu (1966, 1970), Petrescu (1993, 1995), Petrescu et al. (2004), Gache (2005), Bănică $(2004,2007,2008)$ etc. 
The migration of birds, generally, and the migration of diurnal raptors over the Black Sea, have been however poorly documented over time, with only a few articles dealing with this subject in scientific literature.

The first study dealing with bird migration over the Black Sea was the one of Drost (1930), who made a one-month expedition (from the $15^{\text {th }}$ of April to the $13^{\text {th }}$ of May 1928) on the Snakes Island in order to make observations regarding bird spring migration. On the day of arrival, the $15^{\text {th }}$ of April, 1928 he reported 23 bird species and that this number was "not a fair average but a minimum". The number of species observed daily increased 3 times, and on the $10^{\text {th }}$ of May 1928, it has risen to 74 . During all this period, on the island there was a substantial bird migration. He carries out an ornithological inventory of 142 migratory species and two nesting bird species.

In the autumn of 2006, two ornithologists made independent, but concurrent observations on the Black Sea, Calder \& Calder (2006) and Cârnat (2006). On the $4^{\text {th }}$ of October 2006, between the cities of Trabzon (Turkey) and Batumi (Georgia), Calder observed thousands of birds flying around the vessel on which he traveled at night, and hundreds of birds which were resting on the vessel, occupying all places on which they could rest, but also the decks of the ship. The wind blew from East to West at a speed of over $20 \mathrm{~m} / \mathrm{s}$ when it reached the port of Batumi. After sunrise, he made a complete inventory of all species remaining on board, identifying 17 bird species, including a group of 25 Hobbies (Falco subbuteo).

From the same year, some observations on birds over the Black Sea, collected between 2005 and 2006 and on the $7^{\text {th }}$ of October 2006, where publish by Cârnat (op. cit.), who observed an impressive number of about 12,000 passerines flying beside the oil rig where he worked, heading for the Histria-Vadu area, in the East-West direction. The birds were observed between 5:30 and 6:30 AM (local time) and he noticed the very low height of the migration. He managed to identify a number of 12 species, of those that passed nearby. The birds were flying in compact flocks with a density of about 200 specimens per minute.

In 2011, some observations on birds in the southern Black Sea coast of Turkey, consisting in an inventory of 21 species, observed in March 2010, were published by Bell (2011). Finally, the most recent observations were published by Stanciu \& Bănică (2012) who made observations on bird migration in the Black Sea between the $6^{\text {th }}$ and the $14^{\text {th }}$ of April 2008, at variable distance from 5 to $290 \mathrm{~km}$ from the western seashore of the Black Sea.

\section{MATERIAL AND METHODS}

Original observations were collected between 2008-2016 within different scientific expeditions: international scientific Expedition SESAME on the Black Sea (Southern European Seas: Assessing and Modelling Ecosystem Changes), on "Mare Nigrum" research vessel of the National Research and Development Institute for Marine Geology and Geoecology GEOECOMAR, between the $6^{\text {th }}-14^{\text {th }}$ of April 2008; an ornithological assessment project of Oceanographic Research and Marine Environment Protection Society Oceanic-Club, on "Delphis" vessel from the $6^{\text {th }}$ of April to the $8^{\text {th }}$ of May 2013; a vessel survey near Constanța harbor between the $22^{\text {th }}-23^{\text {rd }}$ of September 2014; survey on an oil rig at $200 \mathrm{~km}$ east of Romanian Black Sea shore from March to October 2015; and an ornithological survey on "Ocean Spirit" vessel a Black Sea Oil and Gas company project between the $24^{\text {th }}$ of September to the $11^{\text {th }}$ of November 2016. 
Observations were made taking into account the characteristics of each expedition and two types of methods were used: transect type, in expeditions on research vessels, and vantage point, on oil rigs.

Each event was recorded together with additional information on date, time, number of individuals and behavior of the birds, observation point type, latitude and longitude. All collected data were entered into an electronic database. Additional information, as distance from the nearest seashore were subsequent calculated in ArcGIS 10 ESRI (2011). Field identification of bird species was performed directly or using optical devices: binoculars - Olympus $10 \times 50$, Opticron $7 \times 50$ with integrated compass and DSLR Canon 600D with 100-400 mm Canon lens.

\section{RESULTS AND DISCUSSIONS}

All the original observations of diurnal raptors in the Black Sea were figured with red circle on the maps of each species, together with previous observations from literature, figured with black square if they were older than 1970 and with black triangle if they were newer than 1970 (Fig. 1). The observations from published literature consisted of 132 records, the large majority from Drost (1930) (Tab. 3).

Ten diurnal raptor species have been counted migrating over the Black Sea (Tab. 1). Comparing observations from literature with our data, a total of 13 diurnal raptor species were recorded so far migrating over the Black Sea. Among these, three new species were observed for the first time migrating over the Black Sea: Common Buzzard (Buteo buteo), Hen Harrier (Circus cyaneus) and Eleonora's Falcon (Falco eleonorae). The presence of the Common Buzzard was surprising because the flight characteristics of this species, namely soaring flight using the air currents, do not allow it to make long crossings over the waters.

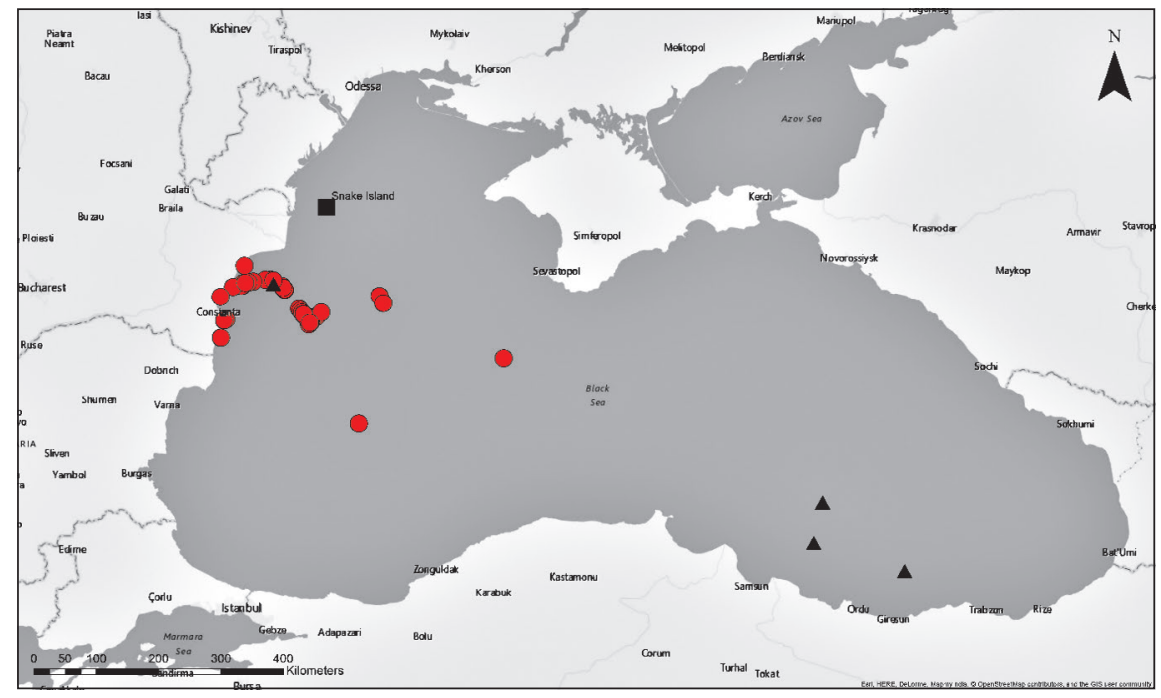

Fig. 1 - Observation points of diurnal raptors in the Black Sea: red circle - original observations; black square - observations from literature older than 1970; black triangle - observations from literature newer than 1970 . 
List of diurnal raptors species observed during migrations over the Black Sea.

Table 1

\begin{tabular}{|r|l|c|c|c|c|c|}
\hline No. & \multicolumn{1}{|c|}{ Species } & Drost (1930) & $\begin{array}{c}\text { Calder \& Calder } \\
(2006)\end{array}$ & Cârnat (2006) & Bell (2011) & present study \\
\hline 1 & Falco peregrinus & $\bullet$ & - & $\bullet$ & - & - \\
\hline 2 & Falco subbuteo & $\bullet$ & $\bullet$ & $\bullet$ & - & $\bullet$ \\
\hline 3 & Falco columbarius & $\bullet$ & - & - & - & $\bullet$ \\
\hline 4 & Falco tinnunculus & $\bullet$ & - & - & $\bullet$ & $\bullet$ \\
\hline 5 & Falco vespertinus & $\bullet$ & - & - & - & - \\
\hline 6 & Falco elenorae & - & - & - & - & $\bullet$ \\
\hline 7 & Circus aeruginosus & $\bullet$ & - & - & - & $\bullet$ \\
\hline 8 & Circus pygargus & $\bullet$ & - & - & - & - \\
\hline 9 & Circus macrourus & $\bullet$ & - & - & - & $\bullet$ \\
\hline 10 & Circus cyaneus & - & - & - & - & $\bullet$ \\
\hline 11 & Accipiter nisus & $\bullet$ & - & - & - & $\bullet$ \\
\hline 12 & Accipiter brevipes & $\bullet$ & - & - & - & $\bullet$ \\
\hline 13 & Buteo buteo & - & - & - & - & $\bullet$ \\
\hline
\end{tabular}

\section{NOTES ON SPECIES}

Buteo buteo - Common Buzzard (Fig. 2). A single individual of this species was observed resting aboard a research vessel in the morning of the $3^{\text {rd }}$ of November, 2016, about $100 \mathrm{~km}$ East of Constanta. The specimen was identified as belonging to the nominate subspecies - Buteo buteo buteo and it was observed at more than 100 $\mathrm{km}$ from the nearest point of the seashore. Referring to the individuals of vulpinus subspecies Moreau (1972) mentions that almost all individuals of this widespread

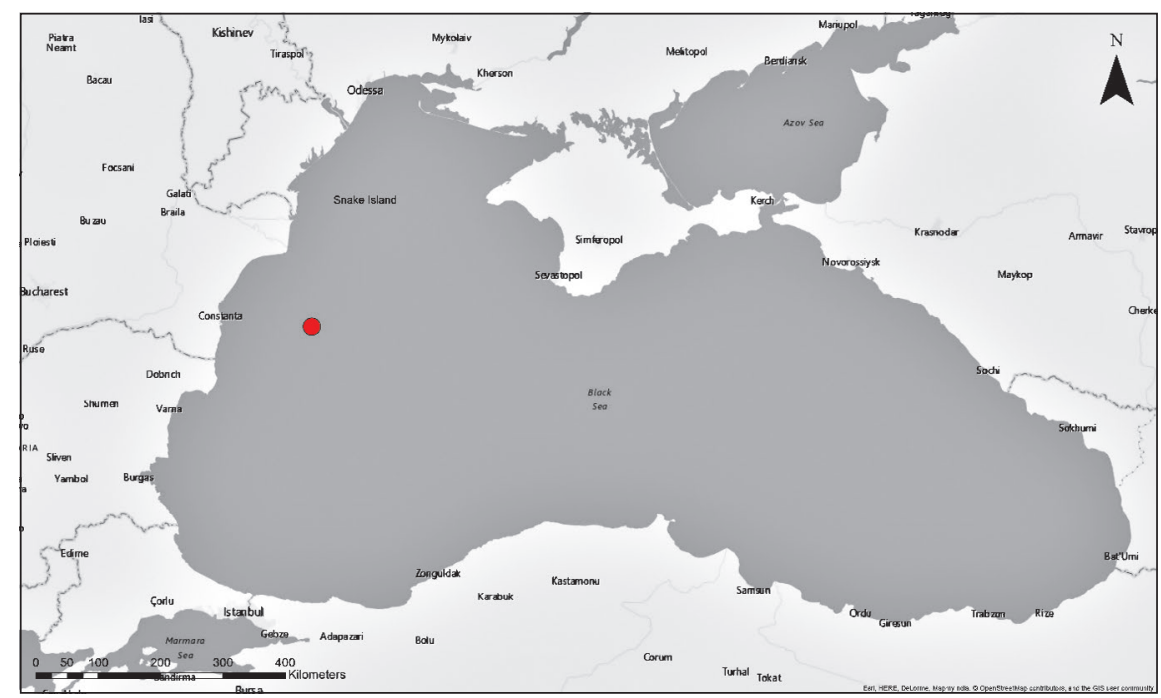

Fig. 2 - Observation points of Common Buzzard - Buteo buteo: red circle - original observations. 
subspecies, which breed from Scandinavia and the Ukraine to about $90^{\circ} \mathrm{E}$, winter in Africa and avoid long sea-crossings. Also, analyzing the migration trends in table 2, it appears that this species avoids crossing the seas over distances of more than 25 $\mathrm{km}$. As far as our observation is concerned, it is remarkable that the bird has ventured so far away from the mainland.

Accipiter brevipes - Levant Sparrowhawk (Fig. 3). Only two records over the Black Sea were made up to now, one on the Snake Island, on the $12^{\text {th }}$ of May 1928 (Drost, 1930), and one on the $23^{\text {rd }}$ of September 2014 offshore Constanța harbor, observed after a storm in the previous day.

Accipiter nisus - Eurasian Sparrowhawk (Fig. 4). The Eurasian Sparrowhawk is one of the two species of raptors with the most observations on the Black Sea, alongside with the Common Kestrel. Analyzing the migration trends in table 2, it appears that this species avoids crossing the seas over distances longer than $100 \mathrm{~km}$. We have recorded individuals of this species up to $200 \mathrm{~km}$ offshore. Individuals were observed many times hunting and feeding on small passerines species which were resting on research vessels or oil rigs. According to the directions of flight from which they arrived or which they left, we believe that individuals of this species flew between vessels and oil rigs, in search of easy food and to rest.

Circus aeruginosus - Marsh Harrier (Fig. 5). Drost (1930) observed several individuals of this species passing over the Snake Island in 15 different days between the $18^{\text {th }}$ of April and the $13^{\text {th }}$ of May 1928. We observed one individual on the $6^{\text {th }}$ of May 2013, in the southern area of the Romanian sea shore, passing from East to West, at a distance of about $10 \mathrm{~km}$ offshore. Characteristics related to migration across water bodies for this species are unknown. Moreau (1972) mentioned that $C$.

Characteristics of raptor migrants observed over the Black Sea (based on Bildstein, 2006).

Table 2

\begin{tabular}{|l|c|c|c|c|c|c|}
\hline \multicolumn{1}{|c|}{ Species } & $\begin{array}{c}\text { Long- } \\
\text { distance } \\
\text { migrant }\end{array}$ & $\begin{array}{c}\text { Trans- } \\
\text { equatorial } \\
\text { migrant }\end{array}$ & $\begin{array}{c}\text { Rains } \\
\text { migrant }\end{array}$ & $\begin{array}{c}\text { Altitudinal } \\
\text { migrant }\end{array}$ & $\begin{array}{c}\text { Maximum } \\
\text { flock size }\end{array}$ & $\begin{array}{c}\text { Maximum distance } \\
\text { traveled over water }\end{array}$ \\
\hline Circus macrourus & $\bullet$ & - & - & - & $<10$ & $<25 \mathrm{~km}$ \\
\hline Circus pygargus & $\bullet$ & $\bullet$ & - & - & $<10$ & $>100 \mathrm{~km}$ \\
\hline Circus cyaneus & $\bullet$ & - & - & - & $<10$ & $>100 \mathrm{~km}$ \\
\hline Accipiter brevipes & $\bullet$ & - & - & - & $>\mathbf{1 0 0 0}$ & $<100 \mathrm{~km}$ \\
\hline Falco vespertinus & $\bullet$ & $\bullet$ & - & - & $>\mathbf{1 0 0 0}$ & $>100 \mathrm{~km}$ \\
\hline Falco eleonorae & $\bullet$ & $\bullet$ & - & - & $>\mathbf{1 0}$ & $>100 \mathrm{~km}$ \\
\hline Falco subbuteo & $\bullet$ & $\bullet$ & $\bullet$ & - & $<\mathbf{1 0 0}$ & $<100 \mathrm{~km}$ \\
\hline Circus aeruginosus & - & - & - & - & & $<100 \mathrm{~km}$ \\
\hline Accipiter nisus & $\bullet$ & - & - & $\bullet$ & $<10$ & $<25 \mathrm{~km}$ \\
\hline Buteo buteo & $\bullet$ & $\bullet$ & - & $\bullet$ & $>\mathbf{1 0 0 0}$ & $<100 \mathrm{~km}$ \\
\hline Falco tinnunculus & - & - & $\bullet$ & - & $<100$ & $>100 \mathrm{~km}$ \\
\hline Falco columbarius & $\bullet$ & - & - & $\bullet$ & $<10$ & $>100 \mathrm{~km}$ \\
\hline Falco peregrinus & $\bullet$ & $\bullet$ & - & - & & $<$ \\
\hline
\end{tabular}




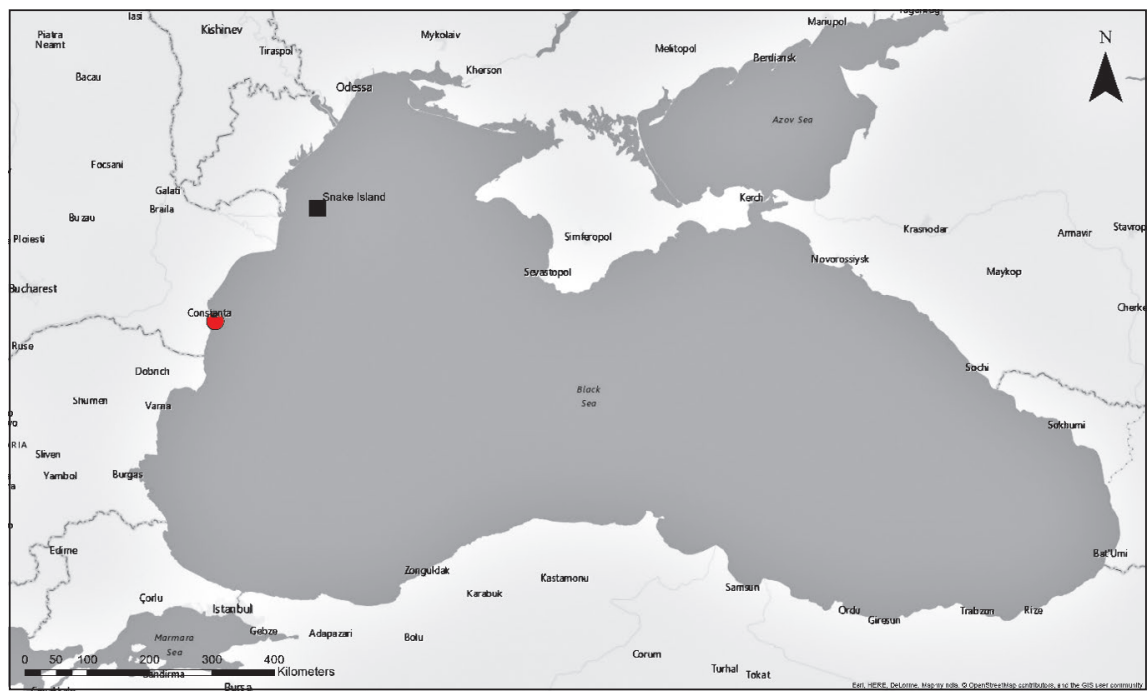

Fig. 3 - Observation points of Levant Sparrowhawk - Accipiter brevipes: red circle - original observations; black square - observations from literature older than 1970.

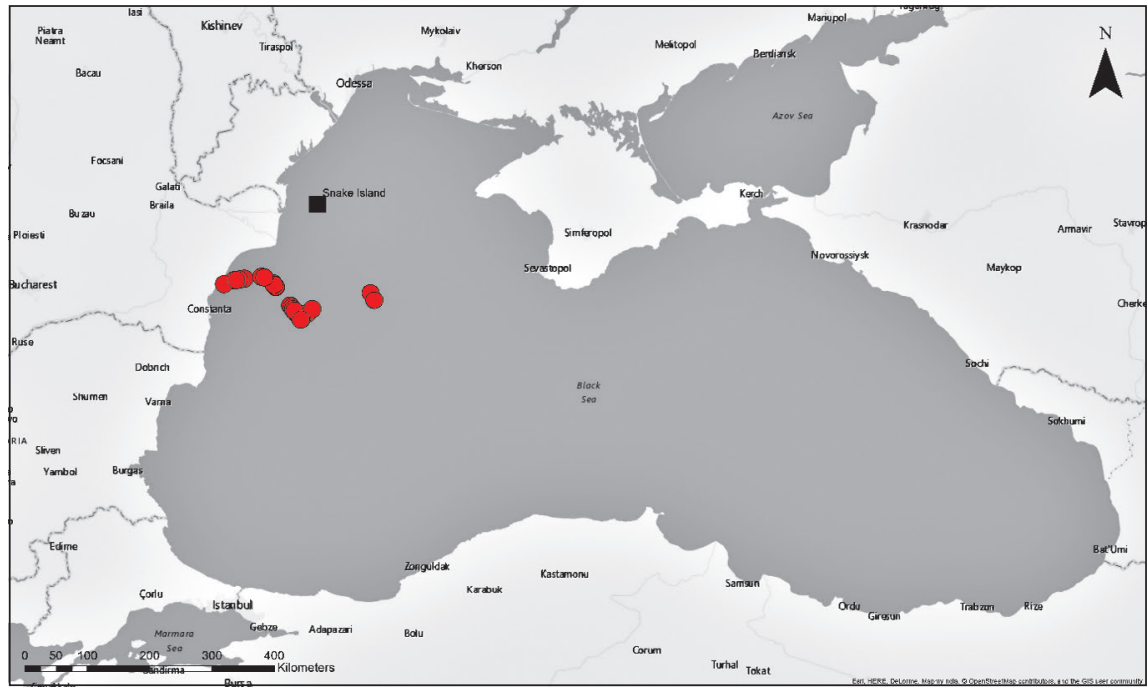

Fig. 4 - Observation points of Eurasian Sparrowhawk - Accipiter nisus: red circle - original observations; black square - observations from literature older than 1970.

aeruginosus does not concentrate on short sea-crossings but it is not known what extent its migration is in fully "broadfront".

Circus cyaneus - Hen Harrier (Fig. 6). On this species of harrier, we made only two observations, both in autumn of 2016: on the $22^{\text {nd }}$ of October and the $30^{\text {th }}$ of October, at distances between $50 \mathrm{~km}$ and $100 \mathrm{~km}$ offshore. It appears that this species 
can transit water bodies over distances longer than $100 \mathrm{~km}$. There is no information in literature on migration across large bodies of water, as this species would migrate across the sea.

Circus macrourus - Pallid Harrier (Fig. 7). Drost (1930) observed several individuals of this species passing over the Snake Island in 22 different days, between the $22^{\text {nd }}$ of April and the $13^{\text {th }}$ of May 1928. We observed one individual on the $21^{\text {st }}$ of October 2016, at a distance of more than $150 \mathrm{~km}$ offshore. Analyzing the migration

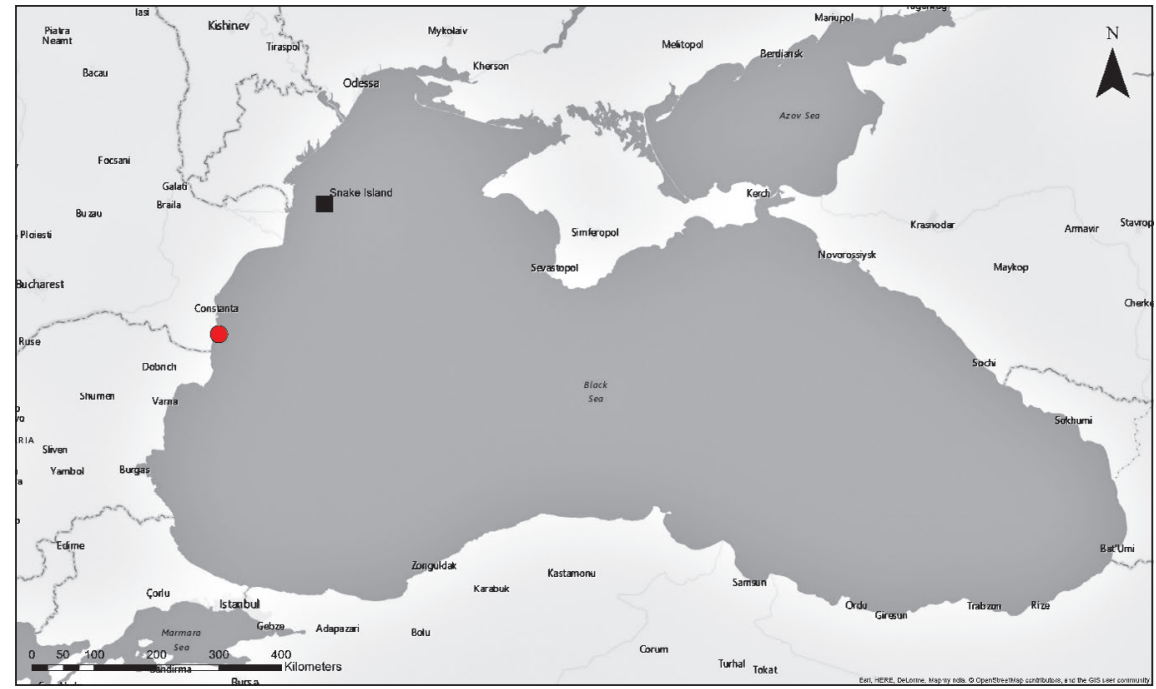

Fig. 5-Observation points of Marsh Harrier - Circus aeruginosus: red circle - original observations; black square - observations from literature older than 1970.

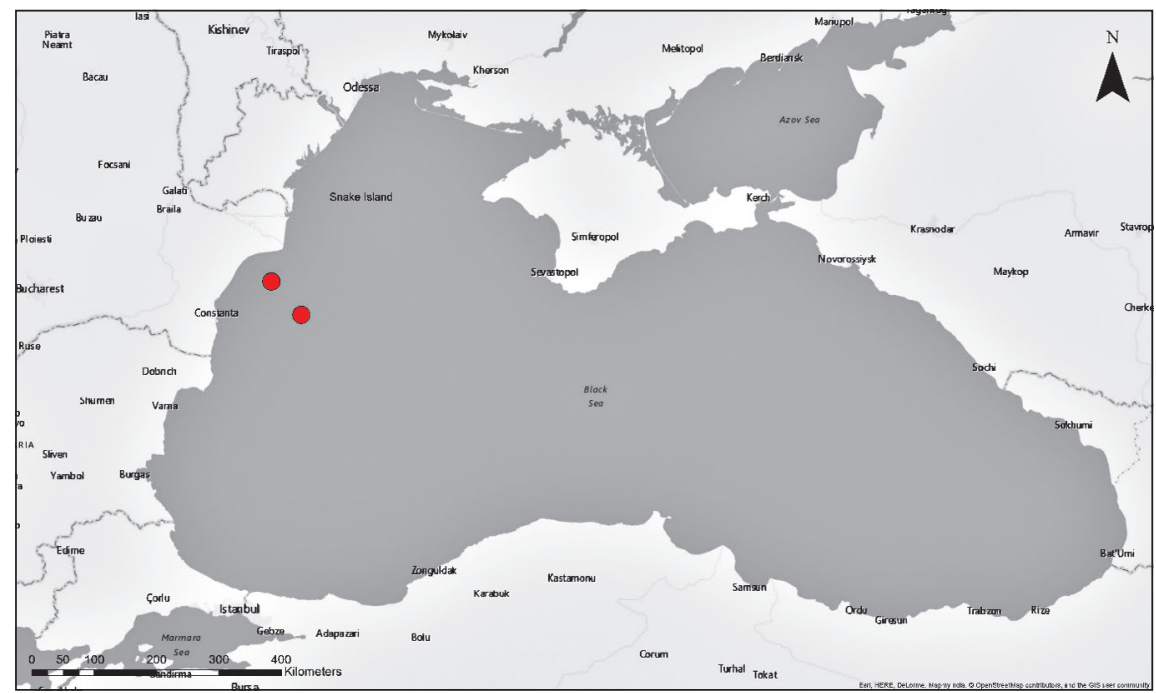

Fig. 6-Observation points of Hen Harrier - Circus cyaneus: red circle - original observations. 
characteristics in table 2, it appears that this species avoids crossing the seas over distances longer than $25 \mathrm{~km}$.

Circus pygargus - Montagu's Harrier. Drost (1930) observed several individuals of this species passing over the Snake Island in 15 different days, during his investigations made there, between the $22^{\text {nd }}$ of April and the $13^{\text {th }}$ of May 1928. From 1928 until now, the species was no longer observed passing over the Black Sea during migration. Analyzing the migration characteristics in table 2, it appears that this species avoids crossing the seas over distances of more than $100 \mathrm{~km}$, the closest Black Sea shore point to Snake Island being about $30 \mathrm{~km}$.

Falco columbarius - Merlin (Fig. 8). Several observations were made on this species, one on the Snake Island, on the $26^{\text {th }}$ of April 1928 (Drost, 1930), and three observations in autumn of 2016, one on the $15^{\text {th }}$ of October and two on the $22^{\text {nd }}$ of October 2016 (one morning and one evening observation), both of them at more than $150 \mathrm{~km}$ from the nearest seashore. Analyzing the migration characteristics in table 2, it appears that this species can transit water bodies over distances longer than 100 $\mathrm{km}$. On the $22^{\text {nd }}$ of October 2016, specimens recorded on the research vessel were observed feeding on small passerines that were resting on the ship. It is possible that individuals of this species, like the Eurasian Sparrow Hawk, fly between ships and marine oil rig, in search of easy food, and resting places.

Falco eleonorae - Eleonora's Falcon (Fig. 9). A single individual of this species was observed on the $30^{\text {th }}$ of April 2013, at about $10 \mathrm{~km}$ offshore. Analyzing the migration characteristics in table 2, it appears that this species can transit water bodies over distances longer than $100 \mathrm{~km}$.

Falco peregrinus - Peregrine Falcon. Drost (1930) observed this species passing over the Snake Island on the $10^{\text {th }}$ of May 1928. Since 1928, the species has

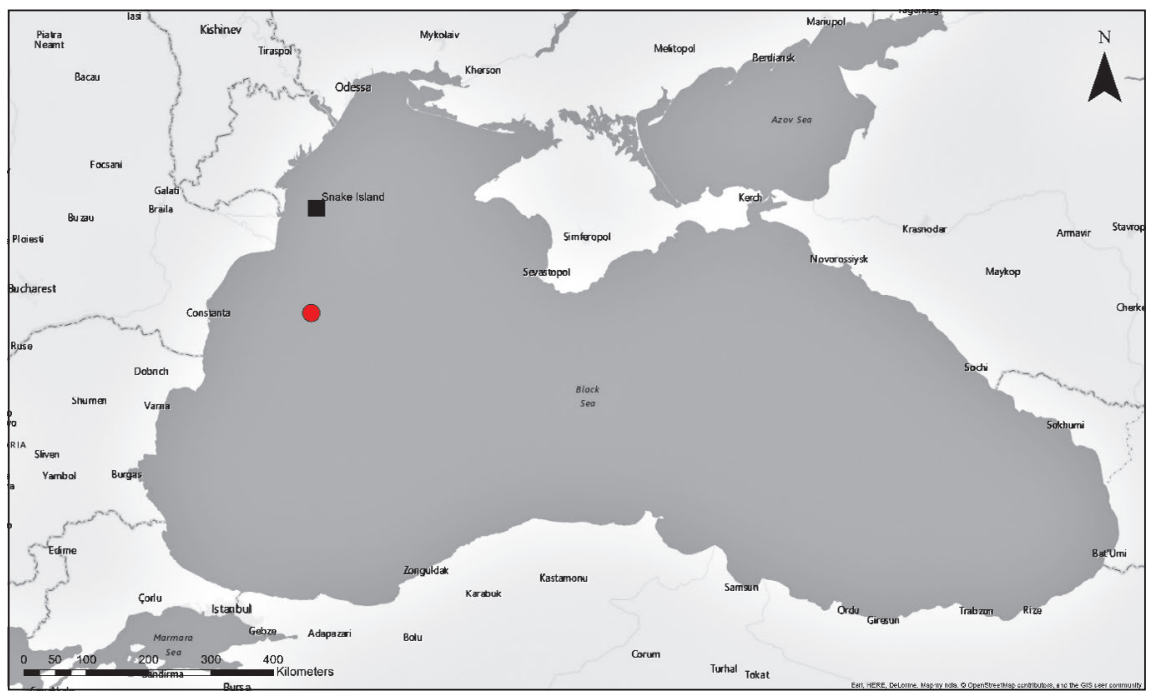

Fig. 7 - Observation points of Pallid Harrier - Circus macrourus: red circle - original observations; black square - observations from literature older than 1970. 


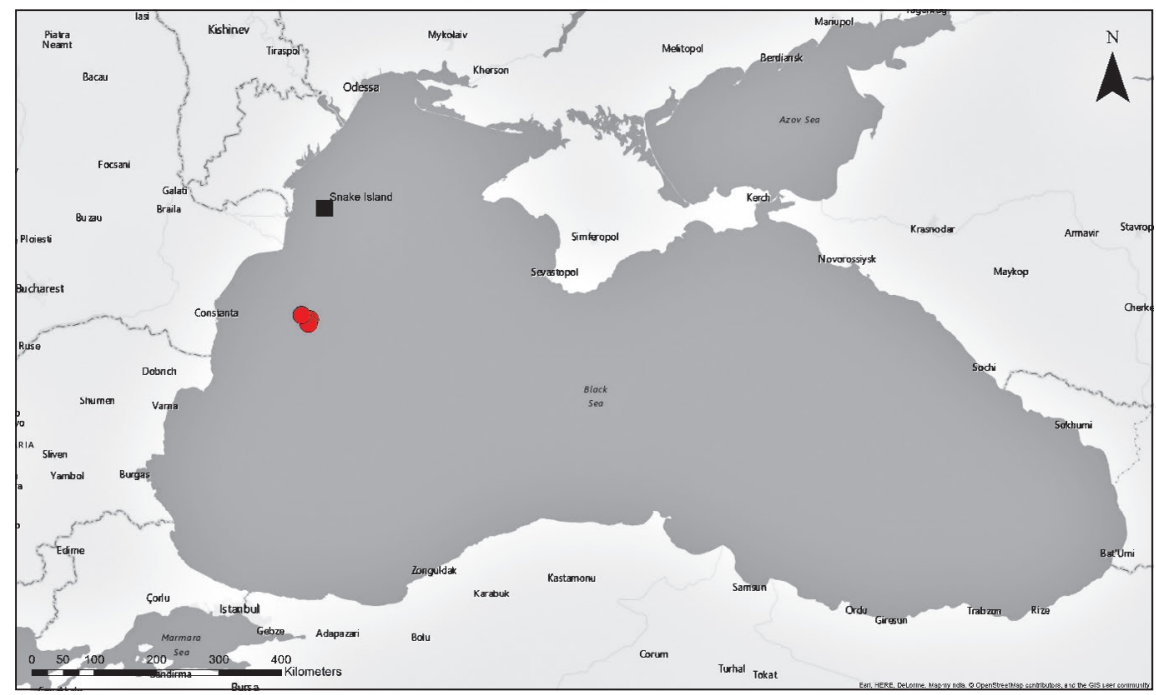

Fig. 8 - Observation points of Merlin - Falco columbarius: red circle - original observations; black square - observations from literature older than 1970.

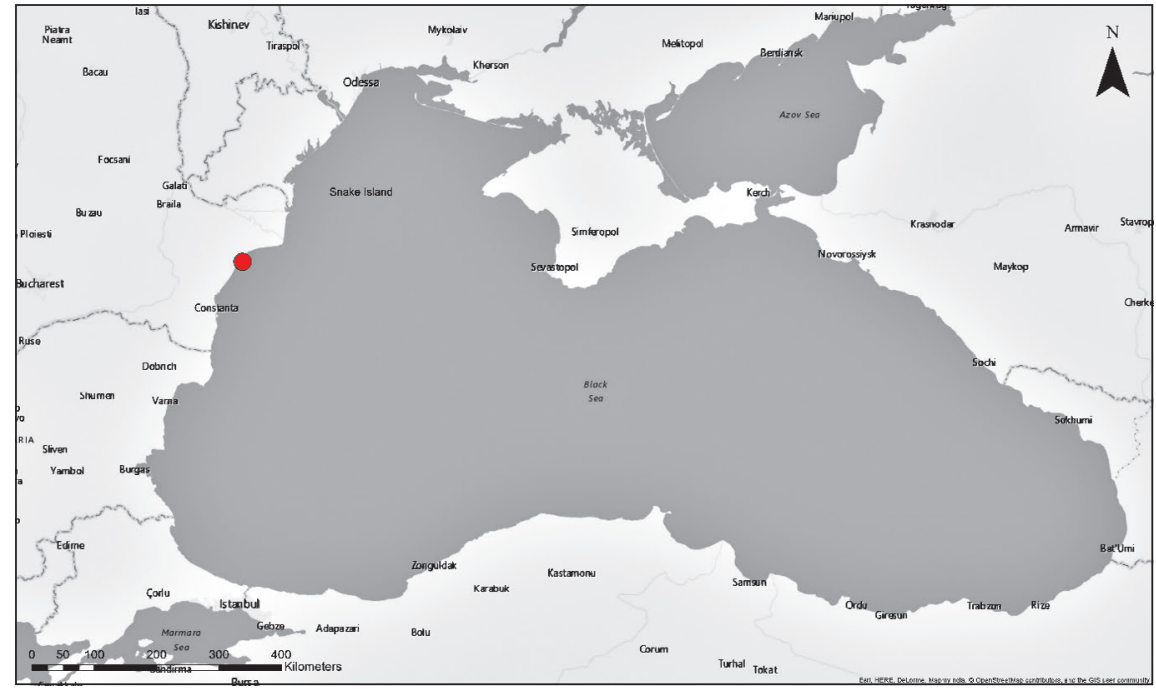

Fig. 9 - Observation points of Eleonora's falcon - Falco eleonorae: red circle - original observations.

been observed only once over the Black Sea in 2006, in the autumn (Cârnat, 2006), when he reported that Falco peregrinus sometimes could be found resting and hunting among the high pillars of oil rigs. Analyzing the migration characteristics in table 2 , it appears that this species can transit water bodies over distances longer than $100 \mathrm{~km}$.

Falco subbuteo - Hobby (Fig. 10). Drost observed numerous individuals of this species passing over the Snake Island in 16 different days between the $18^{\text {th }}$ of April 
and the $13^{\text {th }}$ of May 1928. More recently, Cârnat (2006) observed two individuals between the $12^{\text {th }}-19^{\text {th }}$ of September 2006, resting and feeding on passerines on an oil rig; and Calder \& Calder (2006) who reported 25 specimens observed offshore of Trabzon city (Turkey) resting on the vessel he was traveling with, on the $4^{\text {th }}$ of October 2006. During our survey, we have observed this species four times, on the $22^{\text {nd }}$ and the $23^{\text {rd }}$ of September 2014 in front of Constanța harbor, and on the $8^{\text {th }}$ and $16^{\text {th }}$ of October 2016 offshore, in line with Vadu-Histria area. Analyzing the migration characteristics in table 2, it appears that this species can transit water bodies over distances of less than $100 \mathrm{~km}$.

Falco tinnunculus - Common Kestrel (Fig. 11). The Common Kestrel is the most common species sighted, during the study, among all diurnal raptors, beside the Sparrowhawk. Analyzing the migration characteristics in table 2, it appears that this species avoids crossing the seas over distances longer than $100 \mathrm{~km}$. Drost (1930) observed numerous individuals of this species, passing over the Snake Island in 25 different days, at the time he made observations there, between the $15^{\text {th }}$ of April and the $13^{\text {th }}$ of May 1928. Recently, Bell (2011) observed two migrating individuals on the $8^{\text {th }}$ and the $9^{\text {th }}$ of March 2010, offshore Samsun harbor (Turkey). We have observed the species on numerous occasions, both during the spring and autumn migration, as follows: On the $11^{\text {th }}$ of April 2008 at a distance of about $250 \mathrm{~km}$ from offshore; on $12^{\text {th }}$ of April 2012, about $100 \mathrm{~km}$ South of the Crimean Peninsula; 2 individuals on the $6^{\text {th }}$ of April 2013 at about $10 \mathrm{~km}$ offshore; 3 individuals on the $23^{\text {rd }}$ of September 2014 offshore Constanța harbor; between the $8^{\text {th }}$ of April and the $14^{\text {th }}$ of May 2015 numerous observations on oil rigs, more than $200 \mathrm{~km}$ offshore Romanian shore; between the $28^{\text {th }}$ of September and the $4^{\text {th }}$ of November 2016, we observed four individuals at about $100 \mathrm{~km}$ offshore Romanian coast of the Black Sea.

Our observations show that this species has been present throughout the western Black Sea basin, from distances between 5 and $250 \mathrm{~km}$ from the shoreline.

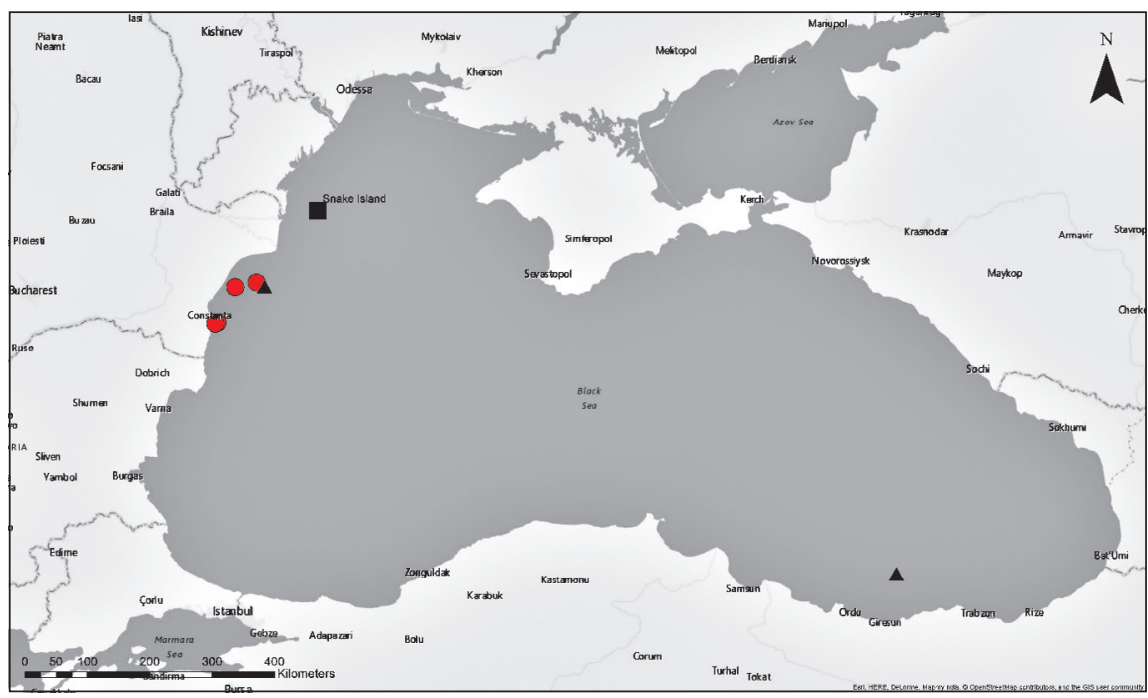

Fig. 10 - Observation points of Hobby - Falco subbuteo: red circle - original observations; black square - observations from literature older than 1970; black triangle - observations from literature newer than 1970. 


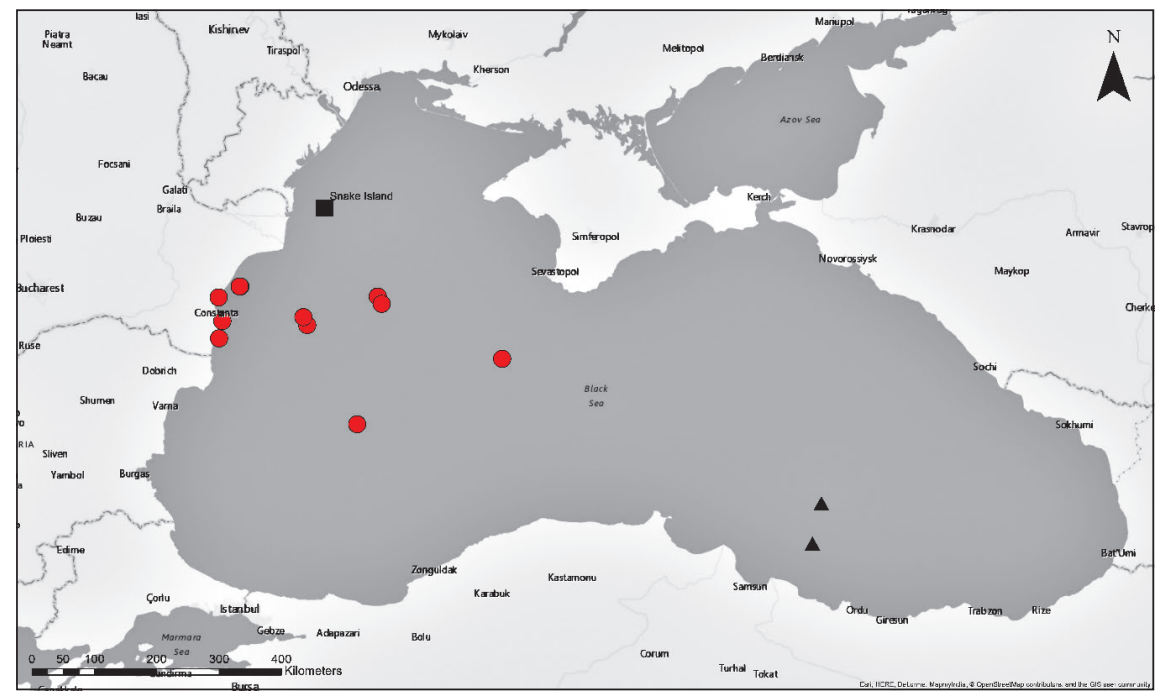

Fig. 11 - Observation points of Common kestrel - Falco tinnunculus: red circle - original observations; black square - observations from literature older than 1970; black triangle - observations from literature newer than 1970 .

It should be mentioned that it is the species that spends the most time on boats or oil rigs. It has often been observed hunting, feeding or resting. It was noticed even roosting on oil rigs or boats, sometimes even several nights in a row. We also believe that this species fly between vessels and marine oil rigs in search of easy food and resting places, considering the flight directions from which they arrive or where they leave. Another interesting observation made is that some birds, including Common Kestrel, are transported from shore to the sea, by vessels, a conclusion we have come from when we noticed the appearance of the birds with the arrival of the supply vessels at the oil rigs.

Falco vespertinus - Red-footed Falcon. Drost (1930) observed several individuals of this species passing over the Snake Island in 15 different days, between the $22^{\text {nd }}$ of April and the $13^{\text {th }}$ of May 1928. From 1928 until now the species was no longer recorded passing over the Black Sea during migration. Analyzing the migration characteristics in table 2, it appears that this species can transit water bodies over distances longer than $100 \mathrm{~km}$.

As mentioned above, of all the species observed, only the Common Buzzard is a species that exploits but depends maximum on the air currents in the seasonal movements that it makes from nesting places to the wintering place and back. Concerning other species of raptors, we can say that they do not depend, to a large extent, on air currents, and they travel through active flight, compensating for the lack of favorable currents or the presence of some unfavorable currents by flapping wings.

The characteristics of migration for the 13 diurnal raptor species that were observed migrating over the Black Sea are presented in table 2. There are four types of analyzed features: long-distance migrants are the species in which at least $20 \%$ of the total population migrates more than $1,500 \mathrm{~km}$ one way; trans-equatorial migrants are 
Table 3

List of diurnal raptors species observed during migrations over the Black Sea (literature data).

\begin{tabular}{|c|c|c|c|c|c|c|c|c|c|}
\hline $\begin{array}{l}\text { Crt. } \\
\text { No. }\end{array}$ & Species & $\begin{array}{c}\text { Observation } \\
\text { date }\end{array}$ & Source & $\begin{array}{l}\text { Number of } \\
\text { individuals }\end{array}$ & Behaviour & $\begin{array}{l}\text { Observation } \\
\text { point }\end{array}$ & Latitude & Longitude & $\begin{array}{l}\text { Distance } \\
\text { to the } \\
\text { nearest } \\
\text { seashore } \\
(\mathrm{km})\end{array}$ \\
\hline 1. & Accipiter brevipes & $12 / 05 / 1928$ & Drost (1930) & 1 & in flight & Snake Island & $45^{\circ} 15^{\prime} 10.793^{\prime \prime}$ & $30^{\circ} 12^{\prime} 23.735^{\prime \prime}$ & 33.3 \\
\hline 2. & Accipiter nisus & $20 / 04 / 1928$ & Drost (1930) & 1 & in flight & Snake Island & $45^{\circ} 15^{\prime} 10.793^{\prime \prime}$ & $30^{\circ} 12^{\prime} 23.735^{\prime \prime}$ & 33.3 \\
\hline 3. & Accipiter nisus & $22 / 04 / 1928$ & Drost (1930) & 1 & in flight & Snake Island & $45^{\circ} 15^{\prime} 10.793^{\prime \prime}$ & $30^{\circ} 12^{\prime} 23.735^{\prime \prime}$ & 102.7 \\
\hline 4. & Accipiter nisus & $24 / 04 / 1928$ & Drost (1930) & 1 & in flight & Snake Island & $45^{\circ} 15^{\prime} 10.793^{\prime \prime}$ & $30^{\circ} 12^{\prime} 23.735^{\prime \prime}$ & 55.1 \\
\hline 5. & Accipiter nisus & $27 / 04 / 1928$ & Drost (1930) & 1 & in flight & Snake Island & $45^{\circ} 15^{\prime} 10.793^{\prime \prime}$ & $30^{\circ} 12^{\prime} 23.735^{\prime \prime}$ & 35.4 \\
\hline 6. & Accipiter nisus & $01 / 05 / 1928$ & Drost (1930) & 30 & in flight & Snake Island & $45^{\circ} 15^{\prime} 10.793^{\prime \prime}$ & $30^{\circ} 12^{\prime} 23.735^{\prime \prime}$ & 35.4 \\
\hline 7. & Accipiter nisus & $02 / 05 / 1928$ & Drost (1930) & 10 & in flight & Snake Island & $45^{\circ} 15^{\prime} 10.793^{\prime \prime}$ & $30^{\circ} 12^{\prime} 23.735^{\prime \prime}$ & 35.4 \\
\hline 8. & Accipiter nisus & $03 / 05 / 1928$ & Drost (1930) & 6 & in flight & Snake Island & $45^{\circ} 15^{\prime} 10.793^{\prime \prime}$ & $30^{\circ} 12^{\prime} 23.735^{\prime \prime}$ & 35.4 \\
\hline 9. & Accipiter nisus & $04 / 05 / 1928$ & Drost (1930) & 2 & in flight & Snake Island & $45^{\circ} 15^{\prime} 10.793$ ' & $30^{\circ} 12^{\prime} 23.735^{\prime \prime}$ & 35.4 \\
\hline 10. & Accipiter nisus & $06 / 05 / 1928$ & Drost (1930) & 1 & in flight & Snake Island & $45^{\circ} 15^{\prime} 10.793^{\prime \prime}$ & $30^{\circ} 12^{\prime} 23.735^{\prime \prime}$ & 35.4 \\
\hline 11. & Accipiter nisus & $07 / 05 / 1928$ & Drost (1930) & 4 & in flight & Snake Island & $45^{\circ} 15^{\prime} 10.793^{\prime \prime}$ & $30^{\circ} 12^{\prime} 23.735^{\prime \prime}$ & 35.4 \\
\hline 12. & Accipiter nisus & $08 / 05 / 1928$ & Drost (1930) & 3 & in flight & Snake Island & $45^{\circ} 15^{\prime} 10.793^{\prime \prime}$ & $30^{\circ} 12^{\prime} 23.735^{\prime \prime}$ & 35.4 \\
\hline 13. & Accipiter nisus & $09 / 05 / 1928$ & Drost (1930) & 1 & in flight & Snake Island & $45^{\circ} 15^{\prime} 10.793^{\prime \prime}$ & $30^{\circ} 12^{\prime} 23.735^{\prime \prime}$ & 35.4 \\
\hline 14. & Accipiter nisus & $10 / 05 / 1928$ & Drost (1930) & 2 & in flight & Snake Island & $45^{\circ} 15^{\prime} 10.793^{\prime \prime}$ & $30^{\circ} 12^{\prime} 23.735^{\prime \prime}$ & 35.4 \\
\hline 15. & Accipiter nisus & $11 / 05 / 1928$ & Drost (1930) & 2 & in flight & Snake Island & $45^{\circ} 15^{\prime} 10.793^{\prime \prime}$ & $30^{\circ} 12^{\prime} 23.735^{\prime \prime}$ & 35.4 \\
\hline 16. & Accipiter nisus & $12 / 05 / 1928$ & Drost (1930) & 2 & in flight & Snake Island & $45^{\circ} 15^{\prime} 10.793^{\prime \prime}$ & $30^{\circ} 12^{\prime} 23.735^{\prime \prime}$ & 35.4 \\
\hline 17. & Accipiter nisus & $13 / 05 / 1928$ & Drost (1930) & 2 & in flight & Snake Island & $45^{\circ} 15^{\prime} 10.793^{\prime \prime}$ & $30^{\circ} 12^{\prime} 23.735^{\prime \prime}$ & 35.4 \\
\hline 18. & Circus aeruginosus & $18 / 04 / 1928$ & Drost (1930) & 1 & in flight & Snake Island & $45^{\circ} 15^{\prime} 10.793^{\prime \prime}$ & $30^{\circ} 12^{\prime} 23.735^{\prime \prime}$ & 35.4 \\
\hline 19. & Circus aeruginosus & $19 / 04 / 1928$ & Drost (1930) & 2 & in flight & Snake Island & $45^{\circ} 15^{\prime} 10.793^{\prime \prime}$ & $30^{\circ} 12^{\prime} 23.735^{\prime \prime}$ & 35.4 \\
\hline 20. & Circus aeruginosus & $20 / 04 / 1928$ & Drost (1930) & 1 & in flight & Snake Island & $45^{\circ} 15^{\prime} 10.793^{\prime \prime}$ & $30^{\circ} 12^{\prime} 23.735^{\prime \prime}$ & 35.4 \\
\hline 21. & Circus aeruginosus & $21 / 04 / 1928$ & Drost (1930) & 1 & in flight & Snake Island & $45^{\circ} 15^{\prime} 10.793^{\prime \prime}$ & $30^{\circ} 12^{\prime} 23.735^{\prime \prime}$ & 35.4 \\
\hline 22. & Circus aeruginosus & $22 / 04 / 1928$ & Drost (1930) & 2 & in flight & Snake Island & $45^{\circ} 15^{\prime} 10.793^{\prime \prime}$ & $30^{\circ} 12^{\prime} 23.735^{\prime \prime}$ & 35.4 \\
\hline 23. & Circus aeruginosus & $23 / 04 / 1928$ & Drost (1930) & 1 & in flight & Snake Island & $45^{\circ} 15^{\prime} 10.793^{\prime \prime}$ & $30^{\circ} 12^{\prime} 23.735^{\prime \prime}$ & 35.4 \\
\hline 24. & Circus aeruginosus & $24 / 04 / 1928$ & Drost (1930) & 2 & in flight & Snake Island & $45^{\circ} 15^{\prime} 10.793^{\prime \prime}$ & $30^{\circ} 12^{\prime} 23.735^{\prime \prime}$ & 35.4 \\
\hline 25. & Circus aeruginosus & $01 / 05 / 1928$ & Drost (1930) & 3 & in flight & Snake Island & $45^{\circ} 15^{\prime} 10.793^{\prime \prime}$ & $30^{\circ} 12^{\prime} 23.735^{\prime \prime}$ & 35.4 \\
\hline 26. & Circus aeruginosus & $02 / 05 / 1928$ & Drost (1930) & 2 & in flight & Snake Island & $45^{\circ} 15^{\prime} 10.793^{\prime \prime}$ & $30^{\circ} 12^{\prime} 23.735^{\prime \prime}$ & 35.4 \\
\hline 27. & Circus aeruginosus & $03 / 05 / 1928$ & Drost (1930) & 2 & in flight & Snake Island & $45^{\circ} 15^{\prime} 10.793^{\prime \prime}$ & $30^{\circ} 12^{\prime} 23.735^{\prime \prime}$ & 35.4 \\
\hline 28. & Circus aeruginosus & $04 / 05 / 1928$ & Drost (1930) & 3 & in flight & Snake Island & $45^{\circ} 15^{\prime} 10.793^{\prime \prime}$ & $30^{\circ} 12^{\prime} 23.735^{\prime \prime}$ & 35.4 \\
\hline 29. & Circus aeruginosus & $05 / 05 / 1928$ & Drost (1930) & 1 & in flight & Snake Island & $45^{\circ} 15^{\prime} 10.793^{\prime \prime}$ & $30^{\circ} 12^{\prime} 23.735^{\prime \prime}$ & 35.4 \\
\hline 30. & Circus aeruginosus & $10 / 05 / 1928$ & Drost (1930) & 3 & in flight & Snake Island & $45^{\circ} 15^{\prime} 10.793^{\prime \prime}$ & $30^{\circ} 12^{\prime} 23.735^{\prime \prime}$ & 35.4 \\
\hline 31. & Circus aeruginosus & $12 / 05 / 1928$ & Drost (1930) & 4 & in flight & Snake Island & $45^{\circ} 15^{\prime} 10.793^{\prime \prime}$ & $30^{\circ} 12^{\prime} 23.735^{\prime \prime}$ & 35.4 \\
\hline 32. & Circus aeruginosus & $13 / 05 / 1928$ & Drost (1930) & 1 & in flight & Snake Island & $45^{\circ} 15^{\prime} 10.793^{\prime \prime}$ & $30^{\circ} 12^{\prime} 23.735^{\prime \prime}$ & 35.4 \\
\hline 33. & Circus macrourus & $22 / 04 / 1928$ & Drost (1930) & 1 & in flight & Snake Island & $45^{\circ} 15^{\prime} 10.793^{\prime \prime}$ & $30^{\circ} 12^{\prime} 23.735^{\prime \prime}$ & 35.4 \\
\hline 34. & Circus macrourus & $23 / 04 / 1928$ & Drost (1930) & 4 & in flight & Snake Island & $45^{\circ} 15^{\prime} 10.793^{\prime \prime}$ & $30^{\circ} 12^{\prime} 23.735^{\prime \prime}$ & 35.4 \\
\hline 35. & Circus macrourus & $24 / 04 / 1928$ & Drost (1930) & 1 & in flight & Snake Island & $45^{\circ} 15^{\prime} 10.793^{\prime \prime}$ & $30^{\circ} 12^{\prime} 23.735^{\prime \prime}$ & 35.4 \\
\hline 36. & Circus macrourus & $25 / 04 / 1928$ & Drost (1930) & 2 & in flight & Snake Island & $45^{\circ} 15^{\prime} 10.793^{\prime \prime}$ & $30^{\circ} 12^{\prime} 23.735^{\prime \prime}$ & 35.4 \\
\hline 37. & Circus macrourus & $26 / 04 / 1928$ & Drost (1930) & 3 & in flight & Snake Island & $45^{\circ} 15^{\prime} 10.793^{\prime \prime}$ & $30^{\circ} 12^{\prime} 23.735^{\prime \prime}$ & 35.4 \\
\hline 38. & Circus macrourus & $27 / 04 / 1928$ & Drost (1930) & 9 & in flight & Snake Island & $45^{\circ} 15^{\prime} 10.793^{\prime \prime}$ & $30^{\circ} 12^{\prime} 23.735^{\prime \prime}$ & 35.4 \\
\hline 39. & Circus macrourus & $28 / 04 / 1928$ & Drost (1930) & 3 & in flight & Snake Island & $45^{\circ} 15^{\prime} 10.793^{\prime \prime}$ & $30^{\circ} 12^{\prime} 23.735^{\prime \prime}$ & 35.4 \\
\hline 40. & Circus macrourus & $29 / 04 / 1928$ & Drost (1930) & 1 & in flight & Snake Island & $45^{\circ} 15^{\prime} 10.793^{\prime \prime}$ & $30^{\circ} 12^{\prime} 23.735^{\prime \prime}$ & 35.4 \\
\hline 41. & Circus macrourus & $30 / 04 / 1928$ & Drost (1930) & 3 & in flight & Snake Island & $45^{\circ} 15^{\prime} 10.793^{\prime \prime}$ & $30^{\circ} 12^{\prime} 23.735^{\prime \prime}$ & 35.4 \\
\hline 42. & Circus macrourus & $01 / 05 / 1928$ & Drost (1930) & 6 & in flight & Snake Island & $45^{\circ} 15^{\prime} 10.793^{\prime \prime}$ & $30^{\circ} 12^{\prime} 23.735^{\prime \prime}$ & 35.4 \\
\hline 43. & Circus macrourus & $02 / 05 / 1928$ & Drost (1930) & 4 & in flight & Snake Island & $45^{\circ} 15^{\prime} 10.793^{\prime \prime}$ & $30^{\circ} 12^{\prime} 23.735^{\prime \prime}$ & 35.4 \\
\hline 44. & Circus macrourus & $03 / 05 / 1928$ & Drost (1930) & 2 & in flight & Snake Island & $45^{\circ} 15^{\prime} 10.793^{\prime \prime}$ & $30^{\circ} 12^{\prime} 23.735^{\prime \prime}$ & 35.4 \\
\hline
\end{tabular}


Table 3 (continued)

\begin{tabular}{|c|c|c|c|c|c|c|c|c|c|}
\hline $\begin{array}{l}\text { Crt. } \\
\text { No. }\end{array}$ & Species & $\begin{array}{l}\text { Observation } \\
\text { date }\end{array}$ & Source & $\begin{array}{l}\text { Number of } \\
\text { individuals }\end{array}$ & Behaviour & $\begin{array}{l}\text { Observation } \\
\text { point }\end{array}$ & Latitude & Longitude & $\begin{array}{c}\text { Distance } \\
\text { to the } \\
\text { nearest } \\
\text { seashore } \\
(\mathrm{km})\end{array}$ \\
\hline 45. & Circus macrourus & $04 / 05 / 1928$ & Drost (1930) & 1 & in flight & Snake Island & $45^{\circ} 15^{\prime} 10.793^{\prime \prime}$ & $30^{\circ} 12^{\prime} 23.735^{\prime \prime}$ & 35.4 \\
\hline 46. & Circus macrourus & 05/05/1928 & Drost (1930) & 1 & in flight & Snake Island & $45^{\circ} 15^{\prime} 10.793^{\prime \prime}$ & $30^{\circ} 12^{\prime} 23.735^{\prime \prime}$ & 35.4 \\
\hline 47. & Circus macrourus & $06 / 05 / 1928$ & Drost (1930) & 2 & in flight & Snake Island & $45^{\circ} 15^{\prime} 10.793^{\prime \prime}$ & $30^{\circ} 12^{\prime} 23.735^{\prime \prime}$ & 35.4 \\
\hline 48. & Circus macrourus & $07 / 05 / 1928$ & Drost (1930) & 1 & in flight & Snake Island & $45^{\circ} 15^{\prime} 10.793^{\prime \prime}$ & $30^{\circ} 12^{\prime} 23.735^{\prime \prime}$ & 35.4 \\
\hline 49. & Circus macrourus & $08 / 05 / 1928$ & Drost (1930) & 1 & in flight & Snake Island & $45^{\circ} 15^{\prime} 10.793^{\prime \prime}$ & $30^{\circ} 12^{\prime} 23.735^{\prime \prime}$ & 35.4 \\
\hline 50. & Circus macrourus & $09 / 05 / 1928$ & Drost (1930) & 1 & in flight & Snake Island & $45^{\circ} 15^{\prime} 10.793^{\prime \prime}$ & $30^{\circ} 12^{\prime} 23.735^{\prime \prime}$ & 35.4 \\
\hline 51. & Circus macrourus & $10 / 05 / 1928$ & Drost (1930) & 2 & in flight & Snake Island & $45^{\circ} 15^{\prime} 10.793^{\prime \prime}$ & $30^{\circ} 12^{\prime} 23.735^{\prime \prime}$ & 35.4 \\
\hline 52. & Circus macrourus & $11 / 05 / 1928$ & Drost (1930) & 1 & in flight & Snake Island & $45^{\circ} 15^{\prime} 10.793^{\prime \prime}$ & $30^{\circ} 12^{\prime} 23.735^{\prime \prime}$ & 35.4 \\
\hline 53. & Circus macrourus & $12 / 05 / 1928$ & Drost (1930) & 2 & in flight & Snake Island & $45^{\circ} 15^{\prime} 10.793^{\prime \prime}$ & $30^{\circ} 12^{\prime} 23.735^{\prime \prime}$ & 35.4 \\
\hline 54. & Circus macrourus & $13 / 05 / 1928$ & Drost (1930) & 1 & in flight & Snake Island & $45^{\circ} 15^{\prime} 10.793^{\prime \prime}$ & $30^{\circ} 12^{\prime} 23.735^{\prime \prime}$ & 35.4 \\
\hline 55. & Circus pygargus & $28 / 04 / 1928$ & Drost (1930) & 1 & in flight & Snake Island & $45^{\circ} 15^{\prime} 10.793^{\prime \prime}$ & $30^{\circ} 12^{\prime} 23.735^{\prime \prime}$ & 35.4 \\
\hline 56. & Circus pygargus & $29 / 04 / 1928$ & Drost (1930) & 1 & in flight & Snake Island & $45^{\circ} 15^{\prime} 10.793^{\prime \prime}$ & $30^{\circ} 12^{\prime} 23.735^{\prime \prime}$ & 35.4 \\
\hline 57. & Circus pygargus & $30 / 04 / 1928$ & Drost (1930) & 1 & in flight & Snake Island & $45^{\circ} 15^{\prime} 10.793^{\prime \prime}$ & $30^{\circ} 12^{\prime} 23.735^{\prime \prime}$ & 35.4 \\
\hline 58. & Circus pygargus & $01 / 05 / 1928$ & Drost (1930) & 2 & in flight & Snake Island & $45^{\circ} 15^{\prime} 10.793^{\prime \prime}$ & $30^{\circ} 12^{\prime} 23.735^{\prime \prime}$ & 35.4 \\
\hline 59. & Circus pygargus & $02 / 05 / 1928$ & Drost (1930) & 1 & in flight & Snake Island & $45^{\circ} 15^{\prime} 10.793^{\prime \prime}$ & $30^{\circ} 12^{\prime} 23.735^{\prime \prime}$ & 35.4 \\
\hline 60. & Circus pygargus & $03 / 05 / 1928$ & Drost (1930) & 2 & in flight & Snake Island & $45^{\circ} 15^{\prime} 10.793^{\prime \prime}$ & $30^{\circ} 12^{\prime} 23.735^{\prime \prime}$ & 35.4 \\
\hline 61. & Circus pygargus & $04 / 05 / 1928$ & Drost (1930) & 2 & in flight & Snake Island & $45^{\circ} 15^{\prime} 10.793^{\prime \prime}$ & $30^{\circ} 12^{\prime} 23.735^{\prime \prime}$ & 35.4 \\
\hline 62. & Circus pygargus & $05 / 05 / 1928$ & Drost (1930) & 1 & in flight & Snake Island & $45^{\circ} 15^{\prime} 10.793^{\prime \prime}$ & $30^{\circ} 12^{\prime} 23.735^{\prime \prime}$ & 35.4 \\
\hline 63. & Circus pygargus & $06 / 05 / 1928$ & Drost (1930) & 2 & in flight & Snake Island & $45^{\circ} 15^{\prime} 10.793^{\prime \prime}$ & $30^{\circ} 12^{\prime} 23.735^{\prime \prime}$ & 35.4 \\
\hline 64. & Circus pygargus & $07 / 05 / 1928$ & Drost (1930) & 1 & in flight & Snake Island & $45^{\circ} 15^{\prime} 10.793^{\prime \prime}$ & $30^{\circ} 12^{\prime} 23.735^{\prime \prime}$ & 35.4 \\
\hline 65. & Circus pygargus & 08/05/1928 & Drost (1930) & 1 & in flight & Snake Island & $45^{\circ} 15^{\prime} 10.793^{\prime \prime}$ & $30^{\circ} 12^{\prime} 23.735^{\prime \prime}$ & 35.4 \\
\hline 66. & Circus pygargus & $09 / 05 / 1928$ & Drost (1930) & 1 & in flight & Snake Island & $45^{\circ} 15^{\prime} 10.793^{\prime \prime}$ & $30^{\circ} 12^{\prime} 23.735^{\prime \prime}$ & 35.4 \\
\hline 67. & Circus pygargus & $10 / 05 / 1928$ & Drost (1930) & 2 & in ingmit & Snake Island & $45^{\circ} 15^{\prime} 10.793^{\prime \prime}$ & $30^{\circ} 12^{\prime} 23.735^{\prime \prime}$ & 35.4 \\
\hline 68. & Circus pygargus & $11 / 05 / 1928$ & Drost (1930) & 1 & in flight & Snake Island & $45^{\circ} 15^{\prime} 10.793^{\prime \prime}$ & $30^{\circ} 12^{\prime} 23.735^{\prime \prime}$ & 35.4 \\
\hline 69. & Circus pygargus & $12 / 05 / 1928$ & Drost (1930) & 1 & in flight & Snake Island & $45^{\circ} 15^{\prime} 10.793^{\prime \prime}$ & $30^{\circ} 12^{\prime} 23.735^{\prime \prime}$ & 35.4 \\
\hline 70. & Falco columbarius & $26 / 04 / 1928$ & Drost (1930) & 1 & in flight & Snake Island & $45^{\circ} 15^{\prime} 10.793^{\prime \prime}$ & $30^{\circ} 12^{\prime} 23.735^{\prime \prime}$ & 35.4 \\
\hline 71. & Falco peregrinus & $19 / 09 / 2006$ & Cârnat (2006) & 1 & feeding & Ship & $44^{\circ} 28^{\prime} 34.673^{\prime \prime}$ & $29^{\circ} 26^{\prime} 36.092^{\prime \prime}$ & 35.4 \\
\hline 72. & Falco peregrinus & $10 / 05 / 1928$ & Drost (1930) & 1 & in flight & Snake Island & $45^{\circ} 15^{\prime} 10.793^{\prime \prime}$ & $30^{\circ} 12^{\prime} 23.735^{\prime \prime}$ & 35.4 \\
\hline 73. & Falco subbuteo & $19 / 09 / 2006$ & Cârnat (2006) & 1 & feeding & Ship & $44^{\circ} 28^{\prime} 34.673^{\prime \prime}$ & $29^{\circ} 26^{\prime} 36.092^{\prime \prime}$ & 35.4 \\
\hline 74. & Falco subbuteo & $18 / 04 / 1928$ & Drost (1930) & 1 & in flight & Snake Island & $45^{\circ} 15^{\prime} 10.793^{\prime \prime}$ & $30^{\circ} 12^{\prime} 23.735^{\prime \prime}$ & 35.4 \\
\hline 75. & Falco subbuteo & $19 / 04 / 1928$ & Drost (1930) & 1 & in flight & Snake Island & $45^{\circ} 15^{\prime} 10.793^{\prime \prime}$ & $30^{\circ} 12^{\prime} 23.735^{\prime \prime}$ & 35.4 \\
\hline 76. & Falco subbuteo & $27 / 04 / 1928$ & Drost (1930) & 2 & in flight & Snake Island & $45^{\circ} 15^{\prime} 10.793^{\prime \prime}$ & $30^{\circ} 12^{\prime} 23.735^{\prime \prime}$ & 35.4 \\
\hline 77. & Falco subbuteo & $28 / 04 / 1928$ & Drost (1930) & 6 & in flight & Snake Island & $45^{\circ} 15^{\prime} 10.793^{\prime \prime}$ & $30^{\circ} 12^{\prime} 23.735^{\prime \prime}$ & 35.4 \\
\hline 78. & Falco subbuteo & $29 / 04 / 1928$ & Drost (1930) & 2 & in flight & Snake Island & $45^{\circ} 15^{\prime} 10.793^{\prime \prime}$ & $30^{\circ} 12^{\prime} 23.735^{\prime \prime}$ & 35.4 \\
\hline 79. & Falco subbuteo & $01 / 05 / 1928$ & Drost (1930) & 10 & in flight & Snake Island & $45^{\circ} 15^{\prime} 10.793^{\prime \prime}$ & $30^{\circ} 12^{\prime} 23.735^{\prime \prime}$ & 35.4 \\
\hline 80. & Falco subbuteo & $02 / 05 / 1928$ & Drost (1930) & 1 & in flight & Snake Island & $45^{\circ} 15^{\prime} 10.793^{\prime \prime}$ & $30^{\circ} 12^{\prime} 23.735^{\prime \prime}$ & 35.4 \\
\hline 81. & Falco subbuteo & 03/05/1928 & Drost (1930) & 5 & in flight & Snake Island & $45^{\circ} 15^{\prime} 10.793^{\prime \prime}$ & $30^{\circ} 12^{\prime} 23.735^{\prime \prime}$ & 35.4 \\
\hline 82. & Falco subbuteo & $04 / 05 / 1928$ & Drost (1930) & 3 & in flight & Snake Island & $45^{\circ} 15^{\prime} 10.793^{\prime \prime}$ & $30^{\circ} 12^{\prime} 23.735^{\prime \prime}$ & 35.4 \\
\hline 83. & Falco subbuteo & 05/05/1928 & Drost (1930) & 1 & in flight & Snake Island & $45^{\circ} 15^{\prime} 10.793^{\prime \prime}$ & $30^{\circ} 12^{\prime} 23.735^{\prime \prime}$ & 35.4 \\
\hline 84. & Falco subbuteo & $06 / 05 / 1928$ & Drost (1930) & 3 & in flight & Snake Island & $45^{\circ} 15^{\prime} 10.793^{\prime \prime}$ & $30^{\circ} 12^{\prime} 23.735^{\prime \prime}$ & 35.4 \\
\hline 85. & Falco subbuteo & 07/05/1928 & Drost (1930) & 2 & in flight & Snake Island & $45^{\circ} 15^{\prime} 10.793^{\prime \prime}$ & $30^{\circ} 12^{\prime} 23.735^{\prime \prime}$ & 35.4 \\
\hline 86. & Falco subbuteo & $10 / 05 / 1928$ & Drost (1930) & 3 & in flight & Snake Island & $45^{\circ} 15^{\prime} 10.793^{\prime \prime}$ & $30^{\circ} 12^{\prime} 23.735^{\prime \prime}$ & 35.4 \\
\hline 87. & Falco subbuteo & $11 / 05 / 1928$ & Drost (1930) & 1 & in flight & Snake Island & $45^{\circ} 15^{\prime} 10.793^{\prime \prime}$ & $30^{\circ} 12^{\prime} 23.735^{\prime \prime}$ & 35.4 \\
\hline 88. & Falco subbuteo & $12 / 05 / 1928$ & Drost (1930) & 2 & in flight & Snake Island & $45^{\circ} 15^{\prime} 10.793^{\prime \prime}$ & $30^{\circ} 12^{\prime} 23.735^{\prime \prime}$ & 35.4 \\
\hline
\end{tabular}


Table 3 (continued)

\begin{tabular}{|c|c|c|c|c|c|c|c|c|c|}
\hline $\begin{array}{l}\text { Crt. } \\
\text { No. }\end{array}$ & Species & $\begin{array}{l}\text { Observation } \\
\text { date }\end{array}$ & Source & $\begin{array}{l}\text { Number of } \\
\text { individuals }\end{array}$ & Behaviour & $\begin{array}{l}\text { Observation } \\
\text { point }\end{array}$ & Latitude & Longitude & $\begin{array}{c}\text { Distance } \\
\text { to the } \\
\text { nearest } \\
\text { seashore } \\
(\mathrm{km})\end{array}$ \\
\hline 89. & Falco subbuteo & $13 / 05 / 1928$ & Drost (1930) & 2 & in flight & Snake Island & $45^{\circ} 15^{\prime} 10.793^{\prime \prime}$ & $30^{\circ} 12^{\prime} 23.735^{\prime \prime}$ & 35.4 \\
\hline 90. & Falco subbuteo & $04 / 10 / 2006$ & Calder (2006) & 25 & on ship & Ship & $41^{\circ} 26^{\prime} 53.380^{\prime \prime}$ & $38^{\circ} 32^{\prime} 39.473^{\prime \prime}$ & 35.4 \\
\hline 91. & Falco tinnunculus & $08 / 03 / 2010$ & Bell (2011) & 1 & feeding & Ship & $42^{\circ} 10^{\prime} 48.000^{\prime \prime}$ & $37^{\circ} 21^{\prime} 43.200^{\prime \prime}$ & 35.4 \\
\hline 92. & Falco tinnunculus & 09/03/2010 & Bell (2011) & 1 & feeding & Ship & $41^{\circ} 45^{\prime} 00.000^{\prime \prime}$ & $37^{\circ} 13^{\prime} 58.800^{\prime \prime}$ & 35.4 \\
\hline 93. & Falco tinnunculus & $15 / 04 / 1928$ & Drost (1930) & 2 & in flight & Snake Island & $45^{\circ} 15^{\prime} 10.793^{\prime \prime}$ & $30^{\circ} 12^{\prime} 23.735^{\prime \prime}$ & 35.4 \\
\hline 94. & Falco tinnunculus & $16 / 04 / 1928$ & Drost (1930) & 3 & in flight & Snake Island & $45^{\circ} 15^{\prime} 10.793^{\prime \prime}$ & $30^{\circ} 12^{\prime} 23.735^{\prime \prime}$ & 35.4 \\
\hline 95. & Falco tinnunculus & $17 / 04 / 1928$ & Drost (1930) & 2 & in flight & Snake Island & $45^{\circ} 15^{\prime} 10.793^{\prime \prime}$ & $30^{\circ} 12^{\prime} 23.735^{\prime \prime}$ & 35.4 \\
\hline 96. & Falco tinnunculus & $18 / 04 / 1928$ & Drost (1930) & 2 & in flight & Snake Island & $45^{\circ} 15^{\prime} 10.793^{\prime \prime}$ & $30^{\circ} 12^{\prime} 23.735^{\prime \prime}$ & 35.4 \\
\hline 97. & Falco tinnunculus & $19 / 04 / 1928$ & Drost (1930) & 2 & in flight & Snake Island & $45^{\circ} 15^{\prime} 10.793^{\prime \prime}$ & $30^{\circ} 12^{\prime} 23.735^{\prime \prime}$ & 35.4 \\
\hline 98. & Falco tinnunculus & $20 / 04 / 1928$ & Drost (1930) & 3 & in flight & Snake Island & $45^{\circ} 15^{\prime} 10.793^{\prime \prime}$ & $30^{\circ} 12^{\prime} 23.735^{\prime \prime}$ & 35.4 \\
\hline 99. & Falco tinnunculus & $21 / 04 / 1928$ & Drost (1930) & 2 & in flight & Snake Island & $45^{\circ} 15^{\prime} 10.793^{\prime \prime}$ & $30^{\circ} 12^{\prime} 23.735^{\prime \prime}$ & 35.4 \\
\hline 100. & Falco tinnunculus & $22 / 04 / 1928$ & Drost (1930) & 3 & in flight & Snake Island & $45^{\circ} 15^{\prime} 10.793^{\prime \prime}$ & $30^{\circ} 12^{\prime} 23.735^{\prime \prime}$ & 35.4 \\
\hline 101. & Falco tinnunculus & $23 / 04 / 1928$ & Drost (1930) & 5 & in flight & Snake Island & $45^{\circ} 15^{\prime} 10.793^{\prime \prime}$ & $30^{\circ} 12^{\prime} 23.735^{\prime \prime}$ & 35.4 \\
\hline 102. & Falco tinnunculus & $26 / 04 / 1928$ & Drost (1930) & 10 & in flight & Snake Island & $45^{\circ} 15^{\prime} 10.793^{\prime \prime}$ & $30^{\circ} 12^{\prime} 23.735^{\prime \prime}$ & 35.4 \\
\hline 103. & Falco tinnunculus & $27 / 04 / 1928$ & Drost (1930) & 7 & in flight & Snake Island & $45^{\circ} 15^{\prime} 10.793^{\prime \prime}$ & $30^{\circ} 12^{\prime} 23.735^{\prime \prime}$ & 35.4 \\
\hline 104. & Falco tinnunculus & $30 / 04 / 1928$ & Drost (1930) & 4 & in flight & Snake Island & $45^{\circ} 15^{\prime} 10.793^{\prime \prime}$ & $30^{\circ} 12^{\prime} 23.735^{\prime \prime}$ & 35.4 \\
\hline 105. & Falco tinnunculus & $01 / 05 / 1928$ & Drost (1930) & 12 & in flight & Snake Island & $45^{\circ} 15^{\prime} 10.793^{\prime \prime}$ & $30^{\circ} 12^{\prime} 23.735^{\prime \prime}$ & 35.4 \\
\hline 106. & Falco tinnunculus & $02 / 05 / 1928$ & Drost (1930) & 17 & in flight & Snake Island & $45^{\circ} 15^{\prime} 10.793^{\prime \prime}$ & $30^{\circ} 12^{\prime} 23.735^{\prime \prime}$ & 35.4 \\
\hline 107. & Falco tinnunculus & 03/05/1928 & Drost (1930) & 7 & in flight & Snake Island & $45^{\circ} 15^{\prime} 10.793$ " & $30^{\circ} 12^{\prime} 23.735^{\prime \prime}$ & 35.4 \\
\hline 108. & Falco tinnunculus & $04 / 05 / 1928$ & Drost (1930) & 4 & in flight & Snake Island & $45^{\circ} 15^{\prime} 10.793^{\prime \prime}$ & $30^{\circ} 12^{\prime} 23.735^{\prime \prime}$ & 35.4 \\
\hline 109. & Falco tinnunculus & 05/05/1928 & Drost (1930) & 1 & in flight & Snake Island & $45^{\circ} 15^{\prime} 10.793^{\prime \prime}$ & $30^{\circ} 12^{\prime} 23.735^{\prime \prime}$ & 35.4 \\
\hline 110. & Falco tinnunculus & $06 / 05 / 1928$ & Drost (1930) & 2 & in flight & Snake Island & $45^{\circ} 15^{\prime} 10.793^{\prime \prime}$ & $30^{\circ} 12^{\prime} 23.735^{\prime \prime}$ & 35.4 \\
\hline 111. & Falco tinnunculus & $07 / 05 / 1928$ & Drost (1930) & 3 & in flight & Snake Island & $45^{\circ} 15^{\prime} 10.793^{\prime \prime}$ & $30^{\circ} 12^{\prime} 23.735^{\prime \prime}$ & 35.4 \\
\hline 112. & Falco tinnunculus & $08 / 05 / 1928$ & Drost (1930) & 4 & in flight & Snake Island & $45^{\circ} 15^{\prime} 10.793^{\prime \prime}$ & $30^{\circ} 12^{\prime} 23.735^{\prime \prime}$ & 35.4 \\
\hline 113. & Falco tinnunculus & 09/05/1928 & Drost (1930) & 2 & in flight & Snake Island & $45^{\circ} 15^{\prime} 10.793^{\prime \prime}$ & $30^{\circ} 12^{\prime} 23.735^{\prime \prime}$ & 35.4 \\
\hline 114. & Falco tinnunculus & $10 / 05 / 1928$ & Drost (1930) & 1 & in flight & Snake Island & $45^{\circ} 15^{\prime} 10.793^{\prime \prime}$ & $30^{\circ} 12^{\prime} 23.735^{\prime \prime}$ & 35.4 \\
\hline 115. & Falco tinnunculus & $11 / 05 / 1928$ & Drost (1930) & 1 & in flight & Snake Island & $45^{\circ} 15^{\prime} 10.793^{\prime \prime}$ & $30^{\circ} 12^{\prime} 23.735^{\prime \prime}$ & 35.4 \\
\hline 116. & Falco tinnunculus & $12 / 05 / 1928$ & Drost (1930) & 2 & in flight & Snake Island & $45^{\circ} 15^{\prime} 10.793^{\prime \prime}$ & $30^{\circ} 12^{\prime} 23.735^{\prime \prime}$ & 35.4 \\
\hline 117. & Falco tinnunculus & $13 / 05 / 1928$ & Drost (1930) & 1 & in flight & Snake Island & $45^{\circ} 15^{\prime} 10.793^{\prime \prime}$ & $30^{\circ} 12^{\prime} 23.735^{\prime \prime}$ & 35.4 \\
\hline 118. & Falco vespertinus & $22 / 04 / 1928$ & Drost (1930) & 1 & in flight & Snake Island & $45^{\circ} 15^{\prime} 10.793^{\prime \prime}$ & $30^{\circ} 12^{\prime} 23.735^{\prime \prime}$ & 35.4 \\
\hline 119. & Falco vespertinus & $23 / 04 / 1928$ & Drost (1930) & 1 & in flight & Snake Island & $45^{\circ} 15^{\prime} 10.793^{\prime \prime}$ & $30^{\circ} 12^{\prime} 23.735^{\prime \prime}$ & 35.4 \\
\hline 120. & Falco vespertinus & $26 / 04 / 1928$ & Drost (1930) & 1 & in flight & Snake Island & $45^{\circ} 15^{\prime} 10.793^{\prime \prime}$ & $30^{\circ} 12^{\prime} 23.735^{\prime \prime}$ & 35.4 \\
\hline 121. & Falco vespertinus & $27 / 04 / 1928$ & Drost (1930) & 1 & in flight & Snake Island & $45^{\circ} 15^{\prime} 10.793^{\prime \prime}$ & $30^{\circ} 12^{\prime} 23.735^{\prime \prime}$ & 35.4 \\
\hline 122. & Falco vespertinus & $29 / 04 / 1928$ & Drost (1930) & 2 & in flight & Snake Island & $45^{\circ} 15^{\prime} 10.793^{\prime \prime}$ & $30^{\circ} 12^{\prime} 23.735^{\prime \prime}$ & 35.4 \\
\hline 123. & Falco vespertinus & $30 / 04 / 1928$ & Drost (1930) & 1 & in flight & Snake Island & $45^{\circ} 15^{\prime} 10.793$ " & $30^{\circ} 12^{\prime} 23.735^{\prime \prime}$ & 35.4 \\
\hline 124. & Falco vespertinus & $01 / 05 / 1928$ & Drost (1930) & 2 & in flight & Snake Island & $45^{\circ} 15^{\prime} 10.793$ ' & $30^{\circ} 12^{\prime} 23.735^{\prime \prime}$ & 35.4 \\
\hline 125. & Falco vespertinus & $02 / 05 / 1928$ & Drost (1930) & 9 & in flight & Snake Island & $45^{\circ} 15^{\prime} 10.793^{\prime \prime}$ & $30^{\circ} 12^{\prime} 23.735^{\prime \prime}$ & 35.4 \\
\hline 126. & Falco vespertinus & $03 / 05 / 1928$ & Drost (1930) & 7 & in flight & Snake Island & $45^{\circ} 15^{\prime} 10.793^{\prime \prime}$ & $30^{\circ} 12^{\prime} 23.735^{\prime \prime}$ & 35.4 \\
\hline 127. & Falco vespertinus & $04 / 05 / 1928$ & Drost (1930) & 5 & in flight & Snake Island & $45^{\circ} 15^{\prime} 10.793^{\prime \prime}$ & $30^{\circ} 12^{\prime} 23.735^{\prime \prime}$ & 35.4 \\
\hline 128. & Falco vespertinus & 05/05/1928 & Drost (1930) & 6 & in flight & Snake Island & $45^{\circ} 15^{\prime} 10.793^{\prime \prime}$ & $30^{\circ} 12^{\prime} 23.735^{\prime \prime}$ & 35.4 \\
\hline 129. & Falco vespertinus & $06 / 05 / 1928$ & Drost (1930) & 3 & in flight & Snake Island & $45^{\circ} 15^{\prime} 10.793^{\prime \prime}$ & $30^{\circ} 12^{\prime} 23.735^{\prime \prime}$ & 35.4 \\
\hline 130. & Falco vespertinus & $07 / 05 / 1928$ & Drost (1930) & 3 & in flight & Snake Island & $45^{\circ} 15^{\prime} 10.793^{\prime \prime}$ & $30^{\circ} 12^{\prime} 23.735^{\prime \prime}$ & 35.4 \\
\hline 131. & Falco vespertinus & $12 / 05 / 1928$ & Drost (1930) & 2 & in flight & Snake Island & $45^{\circ} 15^{\prime} 10.793^{\prime \prime}$ & $30^{\circ} 12^{\prime} 23.735^{\prime \prime}$ & 35.4 \\
\hline 132. & Falco vespertinus & $13 / 05 / 1928$ & Drost (1930) & 1 & in flight & Snake Island & $45^{\circ} 15^{\prime} 10.793$ " & $30^{\circ} 12^{\prime} 23.735^{\prime \prime}$ & 53.6 \\
\hline
\end{tabular}


species that migrate over long distances, at which at least $20 \%$ of the total population migrates across the Equator; rains migrants are species that regularly migrate in response to seasonal rains; altitudinal migrants are species in which at least some populations are known to migrate from high-altitude breeding areas to lower areas during nonbreeding season.

From our observations, the autumn migration of diurnal raptors over the Black Sea runs between the $15^{\text {th }}$ of September and the $10^{\text {th }}$ of November (Fig. 12). The spring migration over the Black Sea runs from the $1^{\text {st }}$ of April to the $15^{\text {th }}$ of May. The highest diurnal activity is between 13:00 - 19:00 (local time), same as Drost observed in 1928 .

We observed that some species spent long time on boats or oil rigs. Falco tinnunculus has been observed on vessels from several hours, up to a few days. This species was observed daily on one oil rig from May until the beginning of August and we assume that it was a single individual, or a couple.

Analyzing Drost's observations in the spring of 1928 and those of autumn 2016 (the longest periods of observations of the Black Sea migration), migration is more intense in spring, raptors travel in small flocks, while autumn migration is less intense, birds traveling alone or at most two.

In the terms of migratory corridors through the western part of the Black Sea basin, we can consider that our observations overlaps on the Western Black Sea Corridor or Via Pontica, as it is well known. Insufficient observations in the East of the Black Sea do not give us a clear picture of the migration in that area, but we can believe that these routes are part of the Trans-Caucasian Corridor. Both corridors are part of the major flyway in Palearctic region, Eurasian-East African Flyway.

In autumn, birds flying from North to South or North-West to South-East are likely to visit first the important bird areas in the North-western Black Sea (Odessa area, Dniprovs'ka Gulf and Tendrivs'ka Gulf), in which the Dniester and Dnieper rivers flow, but also in the Danube Delta area of the Western Black Sea. All of these areas are stopping point and final destination for birds coming from the south during the spring migration. These two major areas are also favorable for the recovery of energy stocks.

The lateral winds towards the migration direction play a significant role in the movement of these birds, having the tendency to divert the birds from their course, thus reaching the sea where they will exploit the upward currents on the small islands (Agostini et al., 2007). This can be one explanation for the large number of raptors observed by Drost in 1928 on the Snake Island in the Black Sea. However, in the absence of these islands (the Snake Island being the only one in the Western Black Sea basin), birds take every opportunity to rest and recover their energy, thus arriving on such occasions to be seen on boats or oil rigs.

In the western part of the Black Sea, we highlighted two routes, one in the Danube Delta, the Dniester and the Dnieper Delta, and another in the Crimean

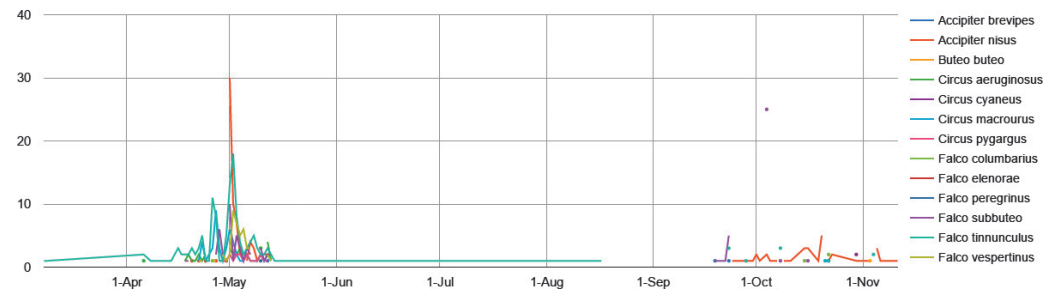

Fig. 12 - Migration periods for diurnal raptors migrating across the Black Sea. 
Table 4

Diurnal raptor observations during migrations over the Black Sea (original observations).

\begin{tabular}{|c|c|c|c|c|c|c|c|c|c|}
\hline $\begin{array}{l}\text { Crt. } \\
\text { No. }\end{array}$ & Species & $\begin{array}{c}\text { Observation } \\
\text { date }\end{array}$ & Time & $\begin{array}{l}\text { Number of } \\
\text { individuals }\end{array}$ & Behaviour & $\begin{array}{l}\text { Observation } \\
\text { point type }\end{array}$ & Latitude & Longitude & $\begin{array}{l}\text { Distance } \\
\text { to the } \\
\text { nearest } \\
\text { seashore } \\
(\mathrm{km})\end{array}$ \\
\hline 1. & Accipiter brevipes & $23 / 09 / 2014$ & & 1 & in flight & Survey ship & $44^{\circ} 5^{\prime} 50.330^{\prime \prime}$ & $28^{\circ} 44^{\prime} 04.650^{\prime \prime}$ & 2.4 \\
\hline 2. & Accipiter nisus & $24 / 09 / 2016$ & 5:53 PM & 1 & in flight & Survey ship & $44^{\circ} 4^{\prime} 15.150^{\prime \prime}$ & $29^{\circ} 58^{\prime} 21.698^{\prime \prime}$ & 86.4 \\
\hline 3. & Accipiter nisus & $30 / 09 / 2016$ & 5:44 PM & 1 & in flight & Survey ship & $44^{\circ} 7^{\prime} 45.088^{\prime \prime}$ & $30^{\circ} 03 ' 27.094^{\prime \prime}$ & 83.6 \\
\hline 4. & Accipiter nisus & $01 / 10 / 2016$ & 2:50 PM & 1 & in flight & Survey ship & $44^{\circ} 12^{\prime} 57.442^{\prime \prime}$ & $29^{\circ} 48^{\prime} 52.751^{\prime \prime}$ & 66.7 \\
\hline 5. & Accipiter nisus & $01 / 10 / 2016$ & 3:52 PM & 1 & in flight & Survey ship & $44^{\circ} 9^{\prime} 49.712^{\prime \prime}$ & $29^{\circ} 52^{\prime} 11.179^{\prime \prime}$ & 73.7 \\
\hline 6. & Accipiter nisus & $02 / 10 / 2016$ & $5: 27 \mathrm{PM}$ & 1 & in flight & Survey ship & $44^{\circ} 8^{\prime} 24.709^{\prime \prime}$ & $29^{\circ} 54^{\prime} 03.560^{\prime \prime}$ & 77.0 \\
\hline 7. & Accipiter nisus & $04 / 10 / 2016$ & 7:33 AM & 1 & in flight & Survey ship & $44^{\circ} 27^{\prime} 12.629^{\prime \prime}$ & $29^{\circ} 34^{\prime} 00.624^{\prime \prime}$ & 35.9 \\
\hline 8. & Accipiter nisus & $04 / 10 / 2016$ & 1:27 PM & 1 & in flight & Survey ship & $44^{\circ} 24^{\prime} 49.313^{\prime \prime}$ & $29^{\circ} 36^{\prime} 38.945^{\prime \prime}$ & 40.9 \\
\hline 9. & Accipiter nisus & $07 / 10 / 2016$ & $6: 15 \mathrm{PM}$ & 1 & in flight & Survey ship & $44^{\circ} 28^{\prime} 59.308^{\prime \prime}$ & $29^{\circ} 00^{\prime} 33.534^{\prime \prime}$ & 11.9 \\
\hline 10. & Accipiter nisus & $09 / 10 / 2016$ & $11: 31 \mathrm{AM}$ & 1 & in flight & Survey ship & $44^{\circ} 26^{\prime} 36.020^{\prime \prime}$ & $28^{\circ} 51^{\prime} 38.970^{\prime \prime}$ & 4.7 \\
\hline 11. & Accipiter nisus & $11 / 10 / 2016$ & 5:54 PM & 1 & in flight & Survey ship & $44^{\circ} 9^{\prime} 08.150^{\prime \prime}$ & $29^{\circ} 53^{\prime} 20.105^{\prime \prime}$ & 75.4 \\
\hline 12. & Accipiter nisus & $15 / 10 / 2016$ & 4:02 PM & 1 & in flight & Survey ship & $44^{\circ} 7^{\prime} 45.728^{\prime \prime}$ & $30^{\circ} 01^{\prime} 46.981^{\prime \prime}$ & 82.5 \\
\hline 13. & Accipiter nisus & $15 / 10 / 2016$ & 4:37 PM & 1 & in flight & Survey ship & $44^{\circ} 6^{\prime} 00.767^{\prime \prime}$ & $29^{\circ} 57^{\prime} 31.957^{\prime \prime}$ & 82.9 \\
\hline 14. & Accipiter nisus & $15 / 10 / 2016$ & 5:31 PM & 1 & in flight & Survey ship & $44^{\circ} 5^{\prime} 43.703^{\prime \prime}$ & $29^{\circ} 56^{\prime} 42.068^{\prime \prime}$ & 83.0 \\
\hline 15. & Accipiter nisus & $16 / 10 / 2016$ & $7: 42 \mathrm{AM}$ & 1 & in flight & Survey ship & $44^{\circ} 31^{\prime} 05.275^{\prime \prime}$ & $29^{\circ} 24^{\prime} 11.736^{\prime \prime}$ & 29.3 \\
\hline 16. & Accipiter nisus & $16 / 10 / 2016$ & $10: 32 \mathrm{AM}$ & 1 & in flight & Survey ship & $44^{\circ} 29^{\prime} 53.279^{\prime \prime}$ & $29^{\circ} 09^{\prime} 27.256^{\prime \prime}$ & 21.7 \\
\hline 17. & Accipiter nisus & $16 / 10 / 2016$ & $11: 27 \mathrm{AM}$ & 1 & in flight & Survey ship & $44^{\circ} 29^{\prime} 25.296^{\prime \prime}$ & $29^{\circ} 05^{\prime} 24.403$ ' & 17.3 \\
\hline 18. & Accipiter nisus & $19 / 10 / 2016$ & $4: 22 \mathrm{PM}$ & 1 & in flight & Survey ship & $44^{\circ} 29^{\prime} 04.783^{\prime \prime}$ & $29^{\circ} 02 ’ 23.017^{\prime \prime}$ & 14.0 \\
\hline 19. & Accipiter nisus & $20 / 10 / 2016$ & 9:30 AM & 2 & in flight & Survey ship & $44^{\circ} 11^{\prime} 59.514^{\prime \prime}$ & $29^{\circ} 50^{\prime} 02.422^{\prime \prime}$ & 68.9 \\
\hline 20. & Accipiter nisus & $20 / 10 / 2016$ & 9:59 AM & 1 & in flight & Survey ship & $44^{\circ} 10^{\prime} 43.745^{\prime \prime}$ & $29^{\circ} 51 ' 22.612^{\prime \prime}$ & 71.7 \\
\hline 21. & Accipiter nisus & $20 / 10 / 2016$ & 3:00 PM & 1 & in flight & Survey ship & $44^{\circ} 4^{\prime} 39.158^{\prime \prime}$ & $29^{\circ} 58^{\prime} 25.136^{\prime \prime}$ & 85.7 \\
\hline 22. & Accipiter nisus & $20 / 10 / 2016$ & 5:27 PM & 1 & in flight & Survey ship & $44^{\circ} 4^{\prime} 41.268^{\prime \prime}$ & $29^{\circ} 57^{\prime} 52.204^{\prime \prime}$ & 85.4 \\
\hline 23. & Accipiter nisus & $22 / 10 / 2016$ & 4:43 PM & 1 & feeding & Survey ship & $44^{\circ} 9^{\prime} 45.299^{\prime \prime}$ & $29^{\circ} 52^{\prime} 26.544^{\prime \prime}$ & 73.9 \\
\hline 24. & Accipiter nisus & $23 / 10 / 2016$ & $7: 18 \mathrm{AM}$ & 1 & feeding & Survey ship & $44^{\circ} 25^{\prime} 01.852^{\prime \prime}$ & $29^{\circ} 36^{\prime} 12.391^{\prime \prime}$ & 40.4 \\
\hline 25. & Accipiter nisus & $23 / 10 / 2016$ & 8:46 AM & 1 & on ship & Survey ship & $44^{\circ} 26^{\prime} 12.905^{\prime \prime}$ & $29^{\circ} 34^{\prime} 57.018^{\prime \prime}$ & 38.0 \\
\hline 26. & Accipiter nisus & $30 / 10 / 2016$ & 11:02 AM & 1 & in flight & Survey ship & $44^{\circ} 30^{\prime} 41.882^{\prime \prime}$ & $29^{\circ} 26^{\prime} 40.438^{\prime \prime}$ & 29.4 \\
\hline 27. & Accipiter nisus & $01 / 11 / 2016$ & 1:42 PM & 1 & in flight & Survey ship & $44^{\circ} 10^{\prime} 50.941^{\prime \prime}$ & $30^{\circ} 08^{\prime} 03.196^{\prime \prime}$ & 82.1 \\
\hline 28. & Accipiter nisus & $03 / 11 / 2016$ & 3:47 PM & 1 & in flight & Survey ship & $44^{\circ} 4^{\prime} 18.001^{\prime \prime}$ & $29^{\circ} 58^{\prime} 10.916^{\prime \prime}$ & 86.2 \\
\hline 29. & Accipiter nisus & $05 / 11 / 2016$ & $7: 31 \mathrm{AM}$ & 2 & feeding & Survey ship & $44^{\circ} 4^{\prime} 17.764^{\prime \prime}$ & $29^{\circ} 58^{\prime} 11.856^{\prime \prime}$ & 86.2 \\
\hline 30. & Accipiter nisus & $05 / 11 / 2016$ & $10: 23 \mathrm{AM}$ & 1 & feeding & Survey ship & $44^{\circ} 4^{\prime} 17.616^{\prime \prime}$ & $29^{\circ} 58^{\prime} 11.809^{\prime \prime}$ & 86.2 \\
\hline 31. & Accipiter nisus & $06 / 11 / 2016$ & 8:19 AM & 1 & in flight & Survey ship & $44^{\circ} 4^{\prime} 17.854^{\prime \prime}$ & $29^{\circ} 58^{\prime} 12.400^{\prime \prime}$ & 86.2 \\
\hline 32. & Accipiter nisus & $11 / 11 / 2016$ & 11:40 AM & 1 & in flight & Survey ship & $44^{\circ} 4^{\prime} 18.401^{\prime \prime}$ & $29^{\circ} 58^{\prime} 10.236^{\prime \prime}$ & 86.2 \\
\hline 33. & Accipiter nisus & $03 / 05 / 2015$ & & 1 & in flight & Oil rig & $44^{\circ} 20^{\prime} 53.959^{\prime \prime}$ & $30^{\circ} 58^{\prime} 26.900^{\prime \prime}$ & 121.9 \\
\hline 34. & Accipiter nisus & $28 / 09 / 2015$ & & 1 & in flight & Oil rig & $44^{\circ} 16^{\prime} 25.115^{\prime \prime}$ & $31^{\circ} 01^{\prime} 39.929^{\prime \prime}$ & 129.6 \\
\hline 35. & Accipiter nisus & $29 / 09 / 2015$ & & 1 & in flight & Oil rig & $44^{\circ} 16^{\prime} 25.115^{\prime \prime}$ & $31^{\circ} 01^{\prime} 39.929^{\prime \prime}$ & 129.6 \\
\hline 36. & Accipiter nisus & $05 / 10 / 2015$ & & 1 & in flight & Oil rig & $44^{\circ} 16^{\prime} 25.115^{\prime \prime}$ & $31^{\circ} 01^{\prime} 39.929^{\prime \prime}$ & 129.6 \\
\hline 37. & Accipiter nisus & $06 / 10 / 2015$ & & 1 & in flight & Oil rig & $44^{\circ} 16^{\prime} 25.115^{\prime \prime}$ & $31^{\circ} 01^{\prime} 39.929^{\prime \prime}$ & 129.6 \\
\hline 38. & Buteo buteo & $03 / 11 / 2016$ & $7: 43 \mathrm{AM}$ & 1 & in flight & Survey ship & $44^{\circ} 4^{\prime} 17.054^{\prime \prime}$ & $29^{\circ} 58^{\prime} 11.183^{\prime \prime}$ & 86.2 \\
\hline 39. & Circus aeruginosus & $06 / 04 / 2013$ & & 1 & in flight & Survey ship & $43^{\circ} 55^{\prime} 00.088^{\prime \prime}$ & $28^{\circ} 41^{\prime} 12.275^{\prime \prime}$ & 4.1 \\
\hline 40. & Circus cyaneus & $22 / 10 / 2016$ & 5:19 PM & 1 & in flight & Survey ship & $44^{\circ} 9^{\prime} 40.784^{\prime \prime}$ & $29^{\circ} 52^{\prime} 31.969^{\prime \prime}$ & 74.1 \\
\hline 41. & Circus cyaneus & $30 / 10 / 2016$ & $11: 41 \mathrm{AM}$ & 2 & in flight & Survey ship & $44^{\circ} 30^{\prime} 41.836^{\prime \prime}$ & $29^{\circ} 26^{\prime} 40.495^{\prime \prime}$ & 29.4 \\
\hline 42. & Circus macrourus & $21 / 10 / 2016$ & 2:00 PM & 1 & in flight & Survey ship & $44^{\circ} 10^{\prime} 48.907^{\prime \prime}$ & $30^{\circ} 08^{\prime} 08.390^{\prime \prime}$ & 82.2 \\
\hline 43. & Falco columbarius & $15 / 10 / 2016$ & $4: 18 \mathrm{PM}$ & 1 & in flight & Survey ship & $44^{\circ} 6^{\prime} 57.748^{\prime \prime}$ & $29^{\circ} 59^{\prime} 53.520^{\prime \prime}$ & 82.7 \\
\hline 44. & Falco columbarius & $22 / 10 / 2016$ & $7: 28 \mathrm{AM}$ & 1 & feeding & Survey ship & $44^{\circ} 4^{\prime} 17.666^{\prime \prime}$ & $29^{\circ} 58^{\prime} 33.506^{\prime \prime}$ & 86.4 \\
\hline 45. & Falco columbarius & $22 / 10 / 2016$ & 5:41 PM & 1 & feeding & Survey ship & $44^{\circ} 9^{\prime} 29.894^{\prime \prime}$ & $29^{\circ} 52^{\prime} 41.869^{\prime \prime}$ & 74.5 \\
\hline 46. & Falco elenorae & $30 / 04 / 2013$ & & 1 & in flight & Survey ship & $44^{\circ} 39^{\prime} 36.320^{\prime \prime}$ & $29^{\circ} 01^{\prime} 38.946^{\prime \prime}$ & 3.5 \\
\hline 47. & Falco subbuteo & 08/10/2016 & $10: 59 \mathrm{AM}$ & 1 & in flight & Survey ship & $44^{\circ} 28^{\prime} 10.747^{\prime \prime}$ & $29^{\circ} 01^{\prime} 14.437^{\prime \prime}$ & 13.5 \\
\hline
\end{tabular}


Table 4 (continued)

\begin{tabular}{|c|c|c|c|c|c|c|c|c|c|}
\hline $\begin{array}{l}\text { Crt. } \\
\text { No. }\end{array}$ & Species & $\begin{array}{l}\text { Observation } \\
\text { date }\end{array}$ & Time & $\begin{array}{l}\text { Number of } \\
\text { individuals }\end{array}$ & Behaviour & $\begin{array}{l}\text { Observation } \\
\text { point type }\end{array}$ & Latitude & Longitude & $\begin{array}{c}\text { Distance } \\
\text { to the } \\
\text { nearest } \\
\text { seashore } \\
(\mathrm{km})\end{array}$ \\
\hline 48. & Falco subbuteo & $16 / 10 / 2016$ & $8: 18 \mathrm{AM}$ & 1 & in flight & Survey ship & $44^{\circ} 31^{\prime} 04.652^{\prime \prime}$ & $29^{\circ} 19^{\prime} 39.508^{\prime \prime}$ & 31.3 \\
\hline 49. & Falco subbuteo & $22 / 09 / 2014$ & & 1 & in flight & Survey ship & $44^{\circ} 6^{\prime} 26.273^{\prime \prime}$ & $28^{\circ} 45^{\prime} 46.109^{\prime \prime}$ & 4.4 \\
\hline 50. & Falco subbuteo & $23 / 09 / 2014$ & & 5 & in flight & Survey ship & $44^{\circ} 5^{\prime} 50.330^{\prime \prime}$ & $28^{\circ} 44^{\prime} 04.650^{\prime \prime}$ & 2.4 \\
\hline 51. & Falco tinnunculus & $28 / 09 / 2016$ & 6:30 PM & 1 & in flight & Survey ship & $44^{\circ} 3^{\prime} 16.348^{\prime \prime}$ & $29^{\circ} 57^{\prime} 21.758^{\prime \prime}$ & 87.5 \\
\hline 52. & Falco tinnunculus & $08 / 10 / 2016$ & 1:05 PM & 2 & in flight & Survey ship & $44^{\circ} 27^{\prime} 14.386^{\prime \prime}$ & $28^{\circ} 59^{\prime} 31.913^{\prime \prime}$ & 12.5 \\
\hline 53. & Falco tinnunculus & $08 / 10 / 2016$ & 1:25 PM & 1 & in flight & Survey ship & $44^{\circ} 27^{\prime} 10.476^{\prime \prime}$ & $28^{\circ} 59^{\prime} 05.798^{\prime \prime}$ & 12.0 \\
\hline 54. & Falco tinnunculus & $22 / 10 / 2016$ & 3:47 PM & 1 & in flight & Survey ship & $44^{\circ} 8^{\prime} 09.780^{\prime \prime}$ & $29^{\circ} 54^{\prime} 06.458^{\prime \prime}$ & 77.5 \\
\hline 55. & Falco tinnunculus & $04 / 11 / 2016$ & 7:31 AM & 1 & in flight & Survey ship & $44^{\circ} 20^{\prime} 24.511^{\prime \prime}$ & $28^{\circ} 40^{\prime} 57.410^{\prime \prime}$ & 0.1 \\
\hline 56. & Falco tinnunculus & $11 / 04 / 2008$ & & 1 & feeding & Survey ship & 43¹'05.999"' & $30^{\circ} 40^{\prime} 32.902^{\prime \prime}$ & 177.5 \\
\hline 57. & Falco tinnunculus & $12 / 04 / 2012$ & & 1 & feeding & Survey ship & $43^{\circ} 42^{\prime} 13.979^{\prime \prime}$ & $32^{\circ} 45^{\prime} 45.608^{\prime \prime}$ & 105.8 \\
\hline 58. & Falco tinnunculus & $06 / 04 / 2013$ & & 2 & in flight & Survey ship & $43^{\circ} 55^{\prime} 00.088^{\prime \prime}$ & $28^{\circ} 41^{\prime} 12.275^{\prime \prime}$ & 4.1 \\
\hline 59. & Falco tinnunculus & $23 / 09 / 2014$ & & 3 & in flight & Survey ship & $44^{\circ} 5^{\prime} 50.330^{\prime \prime}$ & $28^{\circ} 44^{\prime} 04.650^{\prime \prime}$ & 2.4 \\
\hline 60. & Falco tinnunculus & $08 / 04 / 2015$ & & 1 & in flight & Oil rig & $44^{\circ} 20^{\prime} 53.959^{\prime \prime}$ & $30^{\circ} 58^{\prime} 26.900^{\prime \prime}$ & 121.9 \\
\hline 61. & Falco tinnunculus & $11 / 04 / 2015$ & & 1 & in flight/on ship & Oil rig & $44^{\circ} 20^{\prime} 53.959^{\prime \prime}$ & $30^{\circ} 58^{\prime} 26.900^{\prime \prime}$ & 121.9 \\
\hline 62. & Falco tinnunculus & $13 / 04 / 2015$ & & 1 & in flight & Oil rig & $44^{\circ} 20^{\prime} 53.959^{\prime \prime}$ & $30^{\circ} 58^{\prime} 26.900^{\prime \prime}$ & 121.9 \\
\hline 63. & Falco tinnunculus & $14 / 04 / 2015$ & & 1 & in flight & Oil rig & $44^{\circ} 20^{\prime} 53.959^{\prime \prime}$ & $30^{\circ} 58^{\prime} 26.900^{\prime \prime}$ & 121.9 \\
\hline 64. & Falco tinnunculus & $24 / 04 / 2015$ & & 1 & in flight & Oil rig & $44^{\circ} 20^{\prime} 53.959^{\prime \prime}$ & $30^{\circ} 58^{\prime} 26.900^{\prime \prime}$ & 121.9 \\
\hline 65. & Falco tinnunculus & $25 / 04 / 2015$ & & 1 & in flight & Oil rig & $44^{\circ} 20^{\prime} 53.959^{\prime \prime}$ & $30^{\circ} 58^{\prime} 26.900^{\prime \prime}$ & 121.9 \\
\hline 66. & Falco tinnunculus & $26 / 04 / 2015$ & & 1 & in flight & Oil rig & $44^{\circ} 20^{\prime} 53.959^{\prime \prime}$ & $30^{\circ} 58^{\prime} 26.900^{\prime \prime}$ & 121.9 \\
\hline 67. & Falco tinnunculus & $27 / 04 / 2015$ & & 1 & in flight & Oil rig & $44^{\circ} 20^{\prime} 53.959^{\prime \prime}$ & $30^{\circ} 58^{\prime} 26.900^{\prime \prime}$ & 121.9 \\
\hline 68. & Falco tinnunculus & $28 / 04 / 2015$ & & 1 & in flight & Oil rig & $44^{\circ} 20^{\prime} 53.959^{\prime \prime}$ & $30^{\circ} 58^{\prime} 26.900^{\prime \prime}$ & 121.9 \\
\hline 69. & Falco tinnunculus & $29 / 04 / 2015$ & & 1 & in flight & Oil rig & $44^{\circ} 20^{\prime} 53.959^{\prime \prime}$ & $30^{\circ} 58^{\prime} 26.900^{\prime \prime}$ & 121.9 \\
\hline 70. & Falco tinnunculus & $30 / 04 / 2015$ & & 1 & in flight & Oil rig & $44^{\circ} 20^{\prime} 53.959^{\prime \prime}$ & $30^{\circ} 58^{\prime} 26.900^{\prime \prime}$ & 121.9 \\
\hline 71. & Falco tinnunculus & $01 / 05 / 2015$ & & 1 & in flight & Oil rig & $44^{\circ} 20^{\prime} 53.959^{\prime \prime}$ & $30^{\circ} 58^{\prime} 26.900^{\prime \prime}$ & 121.9 \\
\hline 72. & Falco tinnunculus & $02 / 05 / 2015$ & & 1 & in flight & Oil rig & $44^{\circ} 20^{\prime} 53.959^{\prime \prime}$ & $30^{\circ} 58^{\prime} 26.900^{\prime \prime}$ & 121.9 \\
\hline 73. & Falco tinnunculus & $03 / 05 / 2015$ & & 1 & in flight & Oil rig & $44^{\circ} 20^{\prime} 53.959^{\prime \prime}$ & $30^{\circ} 58^{\prime} 26.900^{\prime \prime}$ & 121.9 \\
\hline 74. & Falco tinnunculus & $05 / 05 / 2015$ & & 1 & in flight & Oil rig & $44^{\circ} 20^{\prime} 53.959^{\prime \prime}$ & $30^{\circ} 58^{\prime} 26.900^{\prime \prime}$ & 121.9 \\
\hline 75. & Falco tinnunculus & $06 / 05 / 2015$ & & 1 & in flight & Oil rig & $44^{\circ} 20^{\prime} 53.959^{\prime \prime}$ & $30^{\circ} 58^{\prime} 26.900^{\prime \prime}$ & 121.9 \\
\hline 76. & Falco tinnunculus & $07 / 05 / 2015$ & & 1 & in flight & Oil rig & $44^{\circ} 20^{\prime} 53.959^{\prime \prime}$ & $30^{\circ} 58^{\prime} 26.900^{\prime \prime}$ & 121.9 \\
\hline 77. & Falco tinnunculus & $08 / 05 / 2015$ & & 1 & in flight & Oil rig & $44^{\circ} 20^{\prime} 53.959^{\prime \prime}$ & $30^{\circ} 58^{\prime} 26.900^{\prime \prime}$ & 121.9 \\
\hline 78. & Falco tinnunculus & $09 / 05 / 2015$ & & 1 & in flight & Oil rig & $44^{\circ} 20^{\prime} 53.959^{\prime \prime}$ & $30^{\circ} 58^{\prime} 26.900^{\prime \prime}$ & 121.9 \\
\hline 79. & Falco tinnunculus & $10 / 05 / 2015$ & & 1 & in flight & Oil rig & $44^{\circ} 20^{\prime} 53.959^{\prime \prime}$ & $30^{\circ} 58^{\prime} 26.900^{\prime \prime}$ & 121.9 \\
\hline 80. & Falco tinnunculus & $11 / 05 / 2015$ & & 1 & in flight & Oil rig & $44^{\circ} 20^{\prime} 53.959^{\prime \prime}$ & $30^{\circ} 58^{\prime} 26.900^{\prime \prime}$ & 121.9 \\
\hline 81. & Falco tinnunculus & $12 / 05 / 2015$ & & 1 & in flight & Oil rig & $44^{\circ} 20^{\prime} 53.959^{\prime \prime}$ & $30^{\circ} 58^{\prime} 26.900^{\prime \prime}$ & 121.9 \\
\hline 82. & Falco tinnunculus & $13 / 05 / 2015$ & & 1 & in flight & Oil rig & $44^{\circ} 20^{\prime} 53.959^{\prime \prime}$ & $30^{\circ} 58^{\prime} 26.900^{\prime \prime}$ & 121.9 \\
\hline 83. & Falco tinnunculus & $14 / 05 / 2015$ & & 1 & in flight & Oil rig & $44^{\circ} 20^{\prime} 53.959^{\prime \prime}$ & $30^{\circ} 58^{\prime} 26.900^{\prime \prime}$ & 121.9 \\
\hline 84. & Falco tinnunculus & $16 / 05 / 2015$ & & 1 & in flight & Oil rig & $44^{\circ} 20^{\prime} 53.959^{\prime \prime}$ & $30^{\circ} 58^{\prime} 26.900^{\prime \prime}$ & 121.9 \\
\hline 85. & Falco tinnunculus & $18 / 05 / 2015$ & & 1 & in flight & Oil rig & $44^{\circ} 20^{\prime} 53.959^{\prime \prime}$ & $30^{\circ} 58^{\prime} 26.900^{\prime \prime}$ & 121.9 \\
\hline 86. & Falco tinnunculus & $19 / 05 / 2015$ & & 1 & in flight & Oil rig & $44^{\circ} 20^{\prime} 53.959^{\prime \prime}$ & $30^{\circ} 58^{\prime} 26.900^{\prime \prime}$ & 121.9 \\
\hline 87. & Falco tinnunculus & $20 / 05 / 2015$ & & 1 & in flight & Oil rig & $44^{\circ} 20^{\prime} 53.959^{\prime \prime}$ & $30^{\circ} 58^{\prime} 26.900^{\prime \prime}$ & 121.9 \\
\hline 88. & Falco tinnunculus & $21 / 05 / 2015$ & & 1 & in flight & Oil rig & $44^{\circ} 20^{\prime} 53.959^{\prime \prime}$ & $30^{\circ} 58^{\prime} 26.900^{\prime \prime}$ & 121.9 \\
\hline 89. & Falco tinnunculus & $22 / 05 / 2015$ & & 1 & in flight & Oil rig & $44^{\circ} 20^{\prime} 53.959^{\prime \prime}$ & $30^{\circ} 58^{\prime} 26.900^{\prime \prime}$ & 121.9 \\
\hline 90. & Falco tinnunculus & $23 / 05 / 2015$ & & 1 & in flight & Oil rig & $44^{\circ} 20^{\prime} 53.959^{\prime \prime}$ & $30^{\circ} 58^{\prime} 26.900^{\prime \prime}$ & 121.9 \\
\hline 91. & Falco tinnunculus & $24 / 05 / 2015$ & & 1 & in flight & Oil rig & $44^{\circ} 20^{\prime} 53.959^{\prime \prime}$ & $30^{\circ} 58^{\prime} 26.900^{\prime \prime}$ & 121.9 \\
\hline 92. & Falco tinnunculus & $25 / 05 / 2015$ & & 1 & in flight & Oil rig & $44^{\circ} 20^{\prime} 53.959^{\prime \prime}$ & $30^{\circ} 58^{\prime} 26.900^{\prime \prime}$ & 121.9 \\
\hline 93. & Falco tinnunculus & $26 / 05 / 2015$ & & 1 & in flight & Oil rig & $44^{\circ} 20^{\prime} 53.959^{\prime \prime}$ & $30^{\circ} 58^{\prime} 26.900^{\prime \prime}$ & 121.9 \\
\hline 94. & Falco tinnunculus & $27 / 05 / 2015$ & & 1 & in flight & Oil rig & $44^{\circ} 20^{\prime} 53.959^{\prime \prime}$ & $30^{\circ} 58^{\prime} 26.900^{\prime \prime}$ & 121.9 \\
\hline
\end{tabular}


Table 4 (continued)

\begin{tabular}{|c|c|c|c|c|c|c|c|c|c|}
\hline $\begin{array}{l}\text { Crt. } \\
\text { No. }\end{array}$ & Species & $\begin{array}{l}\text { Observation } \\
\text { date }\end{array}$ & Time & $\begin{array}{l}\text { Number of } \\
\text { individuals }\end{array}$ & Behaviour & $\begin{array}{l}\text { Observation } \\
\text { point type }\end{array}$ & Latitude & Longitude & $\begin{array}{c}\text { Distance } \\
\text { to the } \\
\text { nearest } \\
\text { seashore } \\
(\mathrm{km})\end{array}$ \\
\hline 95. & Falco tinnunculus & $28 / 05 / 2015$ & & 1 & in flight & Oil rig & $44^{\circ} 20^{\prime} 53.959^{\prime \prime}$ & $30^{\circ} 58^{\prime} 26.900^{\prime \prime}$ & 121.9 \\
\hline 96. & Falco tinnunculus & $29 / 05 / 2015$ & & 1 & in flight & Oil rig & $44^{\circ} 20^{\prime} 53.959^{\prime \prime}$ & $30^{\circ} 58^{\prime} 26.900^{\prime \prime}$ & 121.9 \\
\hline 97. & Falco tinnunculus & $30 / 05 / 2015$ & & 1 & in flight & Oil rig & $44^{\circ} 20^{\prime} 53.959^{\prime \prime}$ & $30^{\circ} 58^{\prime} 26.900^{\prime \prime}$ & 121.9 \\
\hline 98. & Falco tinnunculus & $31 / 05 / 2015$ & & 1 & in flight & Oil rig & $44^{\circ} 20^{\prime} 53.959^{\prime \prime}$ & $30^{\circ} 58^{\prime} 26.900^{\prime \prime}$ & 121.9 \\
\hline 99. & Falco tinnunculus & $01 / 06 / 2015$ & & 1 & in flight & Oil rig & $44^{\circ} 20^{\prime} 53.959^{\prime \prime}$ & $30^{\circ} 58^{\prime} 26.900^{\prime \prime}$ & 121.9 \\
\hline 100. & Falco tinnunculus & $02 / 06 / 2015$ & & 1 & in flight & Oil rig & $44^{\circ} 20^{\prime} 53.959^{\prime \prime}$ & $30^{\circ} 58^{\prime} 26.900^{\prime \prime}$ & 121.9 \\
\hline 101. & Falco tinnunculus & $03 / 06 / 2015$ & & 1 & in flight & Oil rig & $44^{\circ} 20^{\prime} 53.959^{\prime \prime}$ & $30^{\circ} 58^{\prime} 26.900^{\prime \prime}$ & 121.9 \\
\hline 102. & Falco tinnunculus & $04 / 06 / 2015$ & & 1 & in flight & Oil rig & $44^{\circ} 20^{\prime} 53.959^{\prime \prime}$ & $30^{\circ} 58^{\prime} 26.900^{\prime \prime}$ & 121.9 \\
\hline 103. & Falco tinnunculus & $05 / 06 / 2015$ & & 1 & in flight & Oil rig & $44^{\circ} 20^{\prime} 53.959^{\prime \prime}$ & $30^{\circ} 58^{\prime} 26.900^{\prime \prime}$ & 121.9 \\
\hline 104. & Falco tinnunculus & $06 / 06 / 2015$ & & 1 & in flight & Oil rig & $44^{\circ} 20^{\prime} 53.959^{\prime \prime}$ & $30^{\circ} 58^{\prime} 26.900^{\prime \prime}$ & 121.9 \\
\hline 105 & Falco tinnunculus & $07 / 06 / 2015$ & & 1 & in flight & Oil rig & $44^{\circ} 20^{\prime} 53.959^{\prime \prime}$ & $30^{\circ} 58^{\prime} 26.900^{\prime \prime}$ & 121.9 \\
\hline 106. & Falco tinnunculus & $08 / 06 / 2015$ & & 1 & in flight & Oil rig & $44^{\circ} 20^{\prime} 53.959^{\prime \prime}$ & $30^{\circ} 58^{\prime} 26.900^{\prime \prime}$ & 121.9 \\
\hline 107. & Falco tinnunculus & $10 / 06 / 2015$ & & 1 & in flight & Oil rig & $44^{\circ} 20^{\prime} 53.959^{\prime \prime}$ & $30^{\circ} 58^{\prime} 26.900^{\prime \prime}$ & 121.9 \\
\hline 108. & Falco tinnunculus & $14 / 06 / 2015$ & & 1 & in flight & Oil rig & $44^{\circ} 20^{\prime} 53.959^{\prime \prime}$ & $30^{\circ} 58^{\prime} 26.900^{\prime \prime}$ & 121.9 \\
\hline 109. & Falco tinnunculus & $24 / 06 / 2015$ & & 1 & in flight & Oil rig & $44^{\circ} 20^{\prime} 53.959^{\prime \prime}$ & $30^{\circ} 58^{\prime} 26.900^{\prime \prime}$ & 121.9 \\
\hline 110. & Falco tinnunculus & $04 / 07 / 2015$ & & 1 & in flight & Oil rig & $44^{\circ} 20^{\prime} 53.959^{\prime \prime}$ & $30^{\circ} 58^{\prime} 26.900^{\prime \prime}$ & 121.9 \\
\hline 111. & Falco tinnunculus & $20 / 07 / 2015$ & & 1 & in flight & Oil rig & $44^{\circ} 16^{\prime} 25.115^{\prime \prime}$ & $31^{\circ} 01^{\prime} 39.929^{\prime \prime}$ & 129.6 \\
\hline 112. & Falco tinnunculus & $21 / 07 / 2015$ & & 1 & in flight & Oil rig & $44^{\circ} 16^{\prime} 25.115^{\prime \prime}$ & $31^{\circ} 01^{\prime} 39.929^{\prime \prime}$ & 129.6 \\
\hline 113. & Falco tinnunculus & $08 / 08 / 2015$ & & 1 & in flight & Oil rig & $44^{\circ} 16^{\prime} 25.115^{\prime \prime}$ & $31^{\circ} 01^{\prime} 39.929^{\prime \prime}$ & 129.6 \\
\hline 114. & Falco tinnunculus & $13 / 08 / 2015$ & & 1 & in flight & Oil rig & $44^{\circ} 16^{\prime} 25.115^{\prime \prime}$ & $31^{\circ} 01^{\prime} 39.929^{\prime \prime}$ & 129.6 \\
\hline 115. & Falco tinпипсиlus & $17 / 08 / 2015$ & & 1 & in flight & Oil rig & $44^{\circ} 16^{\prime} 25.115^{\prime \prime}$ & $31^{\circ} 01^{\prime} 39.929^{\prime \prime}$ & 129.6 \\
\hline
\end{tabular}

Peninsula that reach the Turkish straits (Panuccio et al., 2017). In the eastern part of the Black Sea we highlighted only one route that connects the Caucasus Mountains to the same Pontic Mountains in northern Turkey.

From there, the birds continue migrating to the wintering areas through the Anatolian Plateau. The birds that we noticed migrating from North to South in spring migration probably come from the Bosphorus Strait. According to our own observations and literature information, atmospheric conditions can severely influence the migration of birds across the Black Sea, all the previous studies Drost (1930), Cârnat (2006), and Calder \& Calder (2006), observing birds migrating from east to west, a surprising direction, abnormal for the study area.

\section{ACKNOWLEDGEMENTS}

We are thankful to two anonymous reviewers for their valuable comments on improving the manuscript. A major part of this study was carried out with the support from Oceanographic Research and Marine Environment Protection Society Oceanic-Club. The study was partially funded by project no. RO1567-IBB04/2017 from the Institute of Biology Bucharest of Romanian Academy.

\section{REFERENCES}

ALLEON, A. (1886) Memoire sur les oiseaux observées dans la Dobrudja et la Bulgarie. Ornithologische Jahrbuch, Wien, 2: 297-246.

ALMASY, G.V. (1898) Madartani betekintes a roman Dobrudzaba. Aquila, 5 (1-3): 1-206, Budapest. (in Hungarian) 
AGOSTINI, N., M. GUSTIN, C. CARDELLI (2007) Factors shaping pathways of European Honeybuzzards (Pernis apivorus) during spring migration in the central Mediterranean basin. Journal of Raptor Research, 41: 57-61.

BĂNICĂ, G. (2004) The common gull wintering in Constanța Harbour, Annual report of the Royal Naval Birdwatching Society "Sea Swalow", 53: 55.

BĂNICĂ, G. (2007) Migration of the Lesser Black-Backed Gull on the Romania litoral of the Black Sea, Annual report of the Royal Naval Birdwatching Society "Sea Swalow", 56: 55-57.

BĂNICĂ, G. (2008) Dynamics of the aquatic birds from the marine litoral of Constanța town in 1997-1998. Scripta Ornitologica Romaniae, 3: 57-67.

BELL, J. (2011) Black Sea and Mediterranean, March 2010, "Sea Swallow", 60: 71-76.

BILDSTEIN, K.L. (2006) Migrating Raptors of the World: Their Ecology and Conservation, Cornell University Press, $320 \mathrm{pp}$.

CALDER, D., J. CALDER (2006) Migration incident in the Black Sea, October 2005, Annual report of the Royal Naval Birdwatching Society "Sea Swalow" 55:42-45, ISSN 0959-4787.

CÂRNAT, M.J. (2006) Observaţii ornitologice în Marea Neagră, pp. 23. In: Despre Păsări, No. 2, Romanian Ornithological Society Publication. (in Romanian)

CIOCHIA, V. (1969) Rezervaţia naturală "Lacul Agigea". Ocrotirea Naturii, 13, 2: 183-189. Bucureşti. (in Romanian)

CIOCHIA, V. (1970) Sylvia melanocephala melanocephala (Gmelin)(Aves), specie nouă pentru fauna României. Studii și Cercetări de Biologie seria Biologie Animală, 22, 5: 433-435. (in Romanian)

CIOCHIA, V. (1971) Contribuţii la cunoaşterea efectelor poluării cu petrol a Mării Negre şi reflectarea acestora asupra păsărilor care iernează pe litoralul românesc. Peuce. Muzeul "Delta Dunării": 457-466, Tulcea. (in Romanian)

CIOCHIA, V. (1972) Rezervaţia naturală "Lacul Techirghiol” (zona de sud-est). Ocrotirea Naturii, 16, 1: 21-31, Bucuresti. (in Romanian)

DROST, R. (1930) Über den vogelzug auf der Schlangeninsel im Schwarzen Meer. R. Friedländer \& Sohn, Berlin. 43 pp. (in German)

GACHE, C. (2005) Ornithological aspects on the swamp Herghelie - Mangalia (România). Scientific Annals of the Danube Delta Institute for Research and Development, Tulcea, 11: 20-26.

HEINRICH, G. (1927) Dobrudschareise 1925. Journal für Ornithologie, 75. 1: 6-37. (in German)

IMPE, van J. (1968) Neue angabean uber die Romanische Ornithofauna. Le Gerfaut, 59: 139-147. (in German)

IMPE, van J. (1969a) Concentration enorme de Podiceps nigricollis Brehm en Dobroudja Roumanie. Alauda, 37, 1: 78-79. (in French)

IMPE, van J. (1969b) Sur la migrations d'autumne de Larus minutus Pallas dans la sud-est de la Roumanie. Alauda, 37, 1: 79-80. (in French)

IMPE, van J. (1969c) Passage regulier de Puffinus puffinus yelkouan Acerbi et Stercorarius pomarinus (Temminck) le long de la côte Roumaine. Alauda, 37 (2): 163-167. (in French)

KORNIS, K. (1931) Madartani tanulmanyutam a roman Dobrudsza 1928 oszen. Kocsag, 4 (4): 120-140. (in Hungarian)

KUMMERLOEWE, H. (1956) Uerwinterde Zweegrohrdommel an der rumanischen Schwartzeerkuste. Ornithologische Mitteillungen, 8 (1): 134. (in German)

KUMMERLOEWE, H. (1957a) Vogelzug am Schwarzen meer. Bonner Zoologische Beitrage, 8: 3-4. (in German)

KUMMERLOEWE, H. (1957b) Ornithologische beobachtungen im "Zubringerraum" (BulgarischRumanische Schwarzmeerkuste des Bosforzuges). Bonner Zoologische Beitrage, 8: 248-274. (in German)

LICHERDOPOL, P.I. (1900) Excursiuni în Dobrogea. 134 pp. Bucureşti. (in Romanian)

MĂTIEŞ, M. (1986) Les routes de migration des oiseaux en Roumanie. Travaux du Muséum d'Histoire Naturelle "Grigore Antipa", 28: 247-263. (in French)

MOREAU, R.E. (1972) The Palaearctic-African Bird Migration Systems. Academic Press London and New York, $384 \mathrm{pp}$.

MÜLLER, A. (1926) Bericht uber eine Sammelreise in die Dobrudscha und auf die Schlangeninsel. Verhandlungen und Mitteilungen des Siebenbürgischen Vereins für Naturwissenschaften zu Hermannstadt, 77: 11-40. (in German)

MUNTEANU, D. (1966) Ornithologische Beobachtungen an der Rumanischen Schwarzmeerkuste. Egretta, 1: 8-11. (in German)

MUNTEANU, D. (1969) Bird migration in Rumania. Bulletin of the British Ornithologists' Club. 89(2): 33-35. 
MUNTEANU, D. (1970) Date asupra avifaunei litoralului românesc al Mării Negre (Agigea - Techirghiol - Eforie). Lucrările Staţiunii de Cercetări Biologice şi Geografice "Stejarul", 3: 351-369. (in Romanian)

PANUCCIO, M., A. DUCHI, G. LUCIA, N. AGOSTINI (2017) Species-Specific Behaviour of Raptors Migrating Across the Turkish Straits in Relation to Weather and Geography. Ardeola, 64(2): $305-324$.

PAPADOPOL, A. (1955) Contribuţii la cunoaşterea faunei ornitologice a litoralului românesc al Mării Negre şi a lacurilor din lungul litoralului. Natura, 7 (4): 100-108. (in Romanian)

PAPADOPOL, A. (1956) Contribuţii la cunoaşterea faunei ornitologice a litoralului românesc al Mării Negre şi a lacurilor din lungul litoralului. Natura, 8 (6): 116-125. (in Romanian)

PAPADOPOL, A. (1963) Cercetări asupra păsărilor de pe litoralul Mării Negre şi a lacurilor litorale din Dobrogea. Comunicări de Zoologie, 2: 159-181. (in Romanian)

PAPADOPOL, A. (1968) Considerations on the dynamics of some birds connected with Danube and Black Sea shore in Romania. Travaux du Muséum d'Histoire Naturelle "Grigore Antipa", 8: 947-957.

PAPADOPOL, A. (1970) Contribuții la cunoasterea avifaunei din sudul Dobrogei. Ocrotirea Naturii, 14 (1): 49-60. (in Romanian)

PETRESCU, A. (1993) Contributions à la connaissance de la nourriture de faucon crecerelle, Falco tinnunculus (Aves, Falconiformes) pendant la croissance des pousins. Travaux du Muséum d'Histoire Naturelle "Grigore Antipa", 33: 441-451. (in French)

PETRESCU, A. (1995) Recent remarks on the avifauna of the Romanian marine coast during summer (Agigea - Vama Veche). Travaux du Muséum d'Histoire Naturelle "Grigore Antipa”, 35: 627-642.

PETRESCU, A., S. APOSTOL, G. CHIŞAMERA (2004) Hergheliei Marsh - Important Station on the Romanian Littoral of the Black Sea for the bird migration, Travaux du Muséum National d'Histoire Naturelle "Grigore Antipa", 46: 233-249.

RUDESCU, L. (1958) Migraţia păsărilor. Ed. Ştiinţifică, Bucureşti. 152 pp. (in Romanian)

SEEBOHM, W. (1881) Excursion to the Dobrudscha. Mitteillungen der Ornithologischen Vereines in Wien, 5 (11): 86-89.

SIMPSON, W. (1861) A fortnight in the Dobrudscha. Mitteillungen der Ornithologischen Vereines in Wien: $361-374$.

SINTENIS, G. (1877) Zur Ornis der Dobrudscha. Journal für Ornithologie, 25: 59-73. (in German)

SPISS, S. (1932) Uber Vogelzug in Rumanien. Publicaţiile Societăţii Naturaliştilor Români, 11: 45-66. (in German)

SPISS, S. (1933) Am Schwarzen Meer in Herbst. Ornithologisches Monatschrift, 58: 1-2. (in German)

STANCIU, C. R., G. BĂNICĂ (2012) New data concerning the bird migration over the Black Sea pag. 153 In: D. Murariu, C. Adam, G. Chişamera, E. Iorgu, L. Popa, O. Popa, 2012 - Annual Zoological Congress of "Grigore Antipa" Museum, Book of Abstracts 229 pp.

VIELLIARD, J. (1967) Bref commentaries sur l'avifaune de Roumanie. Aquila, 35 (1): 62. (in French)

*** ESRI 2011. ArcGIS Desktop: Release 10. Redlands, CA: Environmental Systems Research Institute. 